\title{
Size-dependent accumulation of particles in lysosomes modulates dendritic cell function through impaired antigen degradation
}

This article was published in the following Dove Press journal:

International Journal of Nanomedicine

13 August 2014

Number of times this article has been viewed

\author{
Emilie Seydoux ${ }^{1,2}$ \\ Barbara Rothen-Rutishauser ${ }^{1,3}$ \\ Izabela M Nita' \\ Sandor Balog 3 \\ Amiq Gazdhar' \\ Philip A Stumbles ${ }^{4,5}$ \\ Alke Petri-Fink ${ }^{3,6}$ \\ Fabian Blank ${ }^{1, *}$ \\ Christophe von Garnier ${ }^{1, *}$ \\ 'Department of Respiratory Medicine, \\ Inselspital, Bern University Hospital, \\ Department of Clinical Research, \\ University of Bern, ${ }^{2}$ Graduate School \\ for Cellular and Biomedical Sciences, \\ University of Bern, Bern, Switzerland; \\ ${ }^{3}$ Adolphe Merkle Institute, University \\ of Fribourg, Fribourg, Switzerland; \\ ${ }^{4}$ School of Veterinary and Life \\ Sciences, Molecular and Biomedical \\ Sciences, Murdoch University, Perth, \\ WA, Australia; ${ }^{5}$ Telethon Kids \\ Institute, Perth, WA, Australia; \\ ${ }^{6}$ Department of Chemistry, University \\ of Fribourg, Fribourg, Switzerland \\ *These authors contributed equally \\ to the manuscript
}

Introduction: Nanosized particles may enable therapeutic modulation of immune responses by targeting dendritic cell (DC) networks in accessible organs such as the lung. To date, however, the effects of nanoparticles on DC function and downstream immune responses remain poorly understood.

Methods: Bone marrow-derived DCs (BMDCs) were exposed in vitro to 20 or 1,000 nm polystyrene (PS) particles. Particle uptake kinetics, cell surface marker expression, soluble protein antigen uptake and degradation, as well as in vitro $\mathrm{CD}^{+}{ }^{+} \mathrm{T}$-cell proliferation and cytokine production were analyzed by flow cytometry. In addition, co-localization of particles within the lysosomal compartment, lysosomal permeability, and endoplasmic reticulum stress were analyzed.

Results: The frequency of PS particle-positive CD11 $\mathrm{c}^{+} / \mathrm{CD} 11 \mathrm{~b}^{+}$BMDCs reached an early plateau after 20 minutes and was significantly higher for $20 \mathrm{~nm}$ than for 1,000 nm PS particles at all time-points analyzed. PS particles did not alter cell viability or modify expression of the surface markers CD11b, CD11c, MHC class II, CD40, and CD86. Although particle exposure did not modulate antigen uptake, $20 \mathrm{~nm}$ PS particles decreased the capacity of BMDCs to degrade soluble antigen, without affecting their ability to induce antigen-specific CD4 ${ }^{+} \mathrm{T}$-cell proliferation. Co-localization studies between PS particles and lysosomes using laser scanning confocal microscopy detected a significantly higher frequency of co-localized $20 \mathrm{~nm}$ particles as compared with their 1,000 nm counterparts. Neither size of PS particle caused lysosomal leakage, expression of endoplasmic reticulum stress gene markers, or changes in cytokines profiles.

Conclusion: These data indicate that although supposedly inert PS nanoparticles did not induce DC activation or alteration in CD4+ T-cell stimulating capacity, $20 \mathrm{~nm}$ (but not 1,000 nm) PS particles may reduce antigen degradation through interference in the lysosomal compartment. These findings emphasize the importance of performing in-depth analysis of DC function when developing novel approaches for immune modulation with nanoparticles.

Keywords: polystyrene particles, nanoparticles, immune modulation, mouse dendritic cells, $\mathrm{CD} 4^{+} \mathrm{T}$-cells

\section{Introduction}

In recent years, intense research has focused on novel clinical applications for nanomaterials in medicine, including the potential use of engineered nanoparticles (NPs) as carriers for therapeutic applications. ${ }^{1}$ However, our knowledge regarding immune responses related to particulate exposure remains incomplete. Before nanocarriers may be considered for future medical applications, an in-depth understanding of $\mathrm{NP}$-immune cell interactions is essential. ${ }^{2}$ Due to their nanoscale size, large surface area, surface reactivity, and chemical composition, NPs interact differently with
Correspondence: Fabian Blank

Respiratory Medicine, Department of Clinical Research, Murtenstrasse 50, 3008 Bern, Switzerland

Tel +4 I 3I 6327634

Fax +41316327594

Email fabian.blank@dkf.unibe.ch 
biological systems compared with larger-sized particles of the same material. ${ }^{3}$ Moreover, interactions of nanomaterials with the immune system are affected by physicochemical properties, such as size, surface coating, charge, or shape. ${ }^{4,5}$ Hence, potential nanocarriers require meticulous characterization to rule out any adverse toxicological and immunological responses prior to utilization in humans. ${ }^{6}$

Dendritic cells (DCs) are the most potent antigen-presenting cells (APCs) in the respiratory tract and are specialized for capture, processing, and presentation of antigens. ${ }^{7,8}$ As key elements in regulating immunity and tolerance, DCs are essential for bridging innate and adaptive immune responses. Consequently, they are often targets for immunotherapeutic approaches. Furthermore, the respiratory tract represents an attractive target organ, given its enormous interface where interaction between DCs and novel inhalable nanocarrier-based vaccines may occur. ${ }^{9,10}$ DCs are heterogeneous and exist as multiple distinct subsets. ${ }^{11}$ Upon activation by antigen capture, cytokines, or lipopolysaccharides (LPS), immature DCs undergo a maturation process into so-called mature DCs. ${ }^{12}$ During this process, co-stimulatory molecules and maturation markers are upregulated, and mature DCs migrate towards regional lymph nodes where they may induce expansion of antigenspecific CD4 ${ }^{+}$T-cells. ${ }^{13,14}$ Given the central importance of DCs in regulating immune responses, it is essential to gain knowledge about phenotypic and functional changes occurring following exposure to different particle sizes and at different stages of DC maturation.

We recently reported that among intra-nasally instilled 20, 50, 100, 200, and 1,000 nm polystyrene (PS) particles, particles with a diameter of $20 \mathrm{~nm}$ were most frequently captured by DCs compared with larger 1,000 nm particles, and only the smallest particles led to increased antigenspecific $\mathrm{CD}^{+}{ }^{+} \mathrm{T}$-cell proliferation in local draining lymph nodes. ${ }^{15}$ For the present study, we utilized similar particles to further investigate basic mechanisms of particle-immune cell interactions and functional changes of DCs in vitro. We hypothesized that particle size is a key element in modulation of DC function (ie, antigen uptake, degradation, and presentation). We employed fluorescent, negatively charged (carboxylated) PS particles of 20 and 1,000 nm in diameter. These widely employed, supposedly inert model particles were chosen as they are readily detected, available in different sizes with a narrow size distribution and were tested to be free of endotoxin contamination. ${ }^{15}$ We herein report our studies investigating the functional effects of 20 and 1,000 nm PS-particle exposure on mouse bone marrow-derived DCs (BMDCs), using in vitro differentiated immature and mature
BMDCs to assess potential immune modulatory properties of PS particles.

\section{Materials and methods Mice}

Female BALB/c and DO11.10 TCR transgenic (BALB/c background) mice were bred specific pathogen free at the Department of Clinical Research (University of Berne, Switzerland) according to the Swiss Federal Veterinary Office guidelines under animal experimentation permission and used at 8-12 weeks of age.

\section{BMDC cultures, maturation, and exposure to PS particles}

DCs were generated from bone marrow as described elsewhere. ${ }^{16}$ This robust protocol provides a mixture of immature and mature DCs in the non-adherent cell fraction. For our experiments, we strictly employed non-adherent cells that are a mixture of DC precursors, immature DCs, and mature DCs. Briefly, BMDCs were generated by culturing in Iscove's Modified Dulbecco's Medium (IMDM) (Invitrogen, Lucerne, Switzerland) containing 10\% fetal calf serum (FCS), 1\% L-glutamine, 1\% penicillin/streptomycin, and $5 \%$ granulocyte-macrophage colony-stimulating factor (GM-CSF) (collected from culture supernatant of a X63-GMCSF myeloma cell line transfected with the murine GM-CSF gene) for 8 days at $37^{\circ} \mathrm{C}$ in a $5 \% \mathrm{CO}_{2}$ humidified incubator. Five percent GM-CSF refers to $5 \%$ of the collected cell media, and titration of GM-CSF was initially performed before experiments with concentrations of $5 \%, 10 \%$, and $15 \%$ GM-CSF. Five percent was then chosen, as it was the lowest concentration capable of inducing BMDC differentiation and activation following LPS incubation (data not shown).

Yellow-green-labeled carboxylated PS particles of $20 \mathrm{~nm}$ (Molecular Probes, Lucerne, Switzerland) or 1,000 nm (Polysciences, Inc., Warrington, PA, USA) were employed. For cell viability experiments, BMDCs were exposed for 16 hours to PS particles at a concentration ranging from $25 \mu \mathrm{g} / \mathrm{mL}$ up to $100 \mu \mathrm{g} / \mathrm{mL}$. For further experiments, concentrations for both PS-particle sizes were adjusted to $25 \mu \mathrm{g} / \mathrm{mL}$ to rule out any cytotoxic effects. For NP-uptake experiments, BMDCs were first matured with $100 \mathrm{ng} / \mathrm{mL}$ LPS (Sigma-Aldrich, Buchs, Switzerland) for 16 hours (overnight), and PS particles were added the following day for different time-points (see below "Particle uptake studies using laser scanning microscopy"). For further experiments (antigen uptake, degradation and presentation experiments, co-localization with lysosomes, and endoplasmic reticulum [ER] stress), PS particles (25 $\mu \mathrm{g} / \mathrm{mL})$ and LPS (100 ng/mL) 
were added concomitantly for 16 hours. All experiments were conducted with a cell concentration of $1 \times 10^{6}$ cells $/ \mathrm{mL}$.

\section{PS-particle characterization}

PS particles were thoroughly characterized prior to use. The hydrodynamic size was measured by dynamic light scattering (DLS) (3DLS Spectrometer; LS Instruments AG, Fribourg, Switzerland). Measurements were performed both in phosphate-buffered saline (PBS) and cell medium employed for experiments (IMDM $+10 \%$ FCS). Zeta potential was determined in PBS using a Brookhaven-90 Plus Particle Size Analyzer (Brookhaven Instruments Corporation, Holtsville, NY, USA). Additionally, all particles were tested endotoxin free (according to the Limulus amebocyte lysate test; Lonza, Walkersville, MD, USA) as already described in a previous publication. ${ }^{15}$

\section{Flow cytometry}

Unless indicated otherwise, all antibodies were purchased from eBioscience (Vienna, Austria). All stainings were performed strictly on ice as described. ${ }^{17}$ BMDC phenotype and activation state were assessed by staining with CD11b-APCAlexa Fluor 780, CD11c-APC, CD40-PE-Cy5, CD86-PECy7, and MHCII-PE. T-cells were stained with CD4-APC or CD4-APC-Alexa Fluor 780, CD3-biotin (secondary antibody: streptavidin-PE-Cy5), CD69-PE-Cy7, DO11.10 TCR (KJ-126)-PE, or DO11.10 TCR (KJ-126)-biotin (secondary antibody: streptavidin-FITC). Relevant isotype control antibodies were employed as indicated. Propidium iodide (Invitrogen) and Annexin V-Alexa647 (BioLegend ${ }^{\circledR}$, Lucerne, Switzerland) staining was performed to measure cell death and apoptosis, respectively. Samples were measured using a SORP LSRII (BD Biosciences, Franklin Lakes, NJ, USA), and the data obtained were analyzed using Flow Jo (Tree Star, Ashland, OR, USA) software.

\section{Particle uptake studies using laser scanning microscopy (LSM)}

BMDCs were matured for 16 hours with LPS, with LPSuntreated BMDCs as controls. All cultures were incubated the following day with 20 or 1,000 nm PS particles $(25 \mu \mathrm{g} / \mathrm{mL})$ for a time course of 10, 20, 40, 60, and 90 minutes, and 2, 4,8 , and 16 hours. After washing, BMDCs were spun down for 6 minutes at $900 \mathrm{rpm}$ onto glass slides (Cytospin ${ }^{\mathrm{TM}}$ cytocentrifuge; Thermo Scientific, Zürich, Switzerland) and fixed in $70 \%$ alcohol for 5 minutes. Cells were permeabilized in $0.2 \%$ Triton X-100 (Sigma-Aldrich) and stained with phalloidin rhodamine (1:100; Molecular Probes) and with DAPI (4',6-diamidino-2-phenylindole; $1 \mu \mathrm{g} / \mathrm{mL}$; Invitro- gen) for 1 hour at room temperature. Afterwards, the cells were washed and mounted in Aquatex mounting medium (Merck KGaA, Darmstadt, Germany). Optical sections were taken with a Zeiss LSM 710 (Carl Zeiss AG, Feldbach, Switzerland) with a $63 \times$ oil objective (Plan-Apochromat $63 \times / 1.40$ Oil DIC M27) and a digital zoom of $3 \times$. Image processing was performed using Imaris ${ }^{\circledR}$ (Bitplane AG, Zurich, Switzerland) software.

\section{Measurement of antigen uptake and antigen degradation}

Prior to antigen uptake, degradation, and presentation studies, ovalbumin (OVA) and bovine serum albumin (BSA) antigens were thoroughly titrated in preliminary experiments using a concentration series of $2.5,10,20$, and $40 \mu \mathrm{g} / \mathrm{mL}$ for uptake and degradation and 5, 10,20,50, and $100 \mu \mathrm{g} / \mathrm{mL}$ for antigen presentation assays. In each case, the smallest concentration of antigen that induced robust $\mathrm{CD} 4{ }^{+} \mathrm{T}$-cell proliferation was chosen (data not shown). Antigen uptake and degradation capacity of BMDCs were assessed as previously described. ${ }^{17,18}$ Briefly, cells were incubated with $2.5 \mu \mathrm{g} / \mathrm{mL}$ OVA-Alexa Fluor 647 (Molecular Probes) to assess uptake capacity or with $10 \mu \mathrm{g} / \mathrm{mL}$ BSA-DQ Red (Invitrogen) to determine degradation capacity. Cells were incubated for a time course of $0-60$ minutes at $37^{\circ} \mathrm{C}$ or on ice (controls).

\section{Stimulation of carboxyfluorescein succinimidyl ester (CFSE)-labeled OVA-specific T-cells}

$\mathrm{CD}^{+}{ }^{+} \mathrm{T}$-cells from BALB/c DO11.10 mice were isolated using a CD4-positive isolation kit (Miltenyi Biotech, Bergisch Gladbach, Germany). Cells were labeled with CFSE (eBioscience) and co-cultured for 72 hours with BMDCs that were previously incubated for 16 hours with LPS and/or PS particles, to a ratio 10:1 (T-cells/DCs). In addition, BMDCs were pulsed for 1 hour with $20 \mu \mathrm{g} / \mathrm{mL}$ OVA protein (Molecular Probes), or $\mathrm{H}-2^{\mathrm{d}}$ restricted OVA peptide $(20 \mu \mathrm{g} / \mathrm{mL}$, sequence $323-339$; GenScript, Aachen, Germany) then added to the co-culture for 72 hours. We employed the non-adherent BMDC cell population without further cell sorting or purification steps. After the incubation period, antigen-specific T-cell proliferation (CFSE dilution) was measured by flow cytometry.

\section{Intracellular cytokine staining}

DO11.10 ${ }^{+} / \mathrm{CD}^{+}{ }^{+} \mathrm{T}$-cells and BMDCs were co-cultured as described in the section "Stimulation of carboxyfluorescein succinimidyl ester (CFSE)-labeled OVA-specific T-cells" with or without $20 \mu \mathrm{g} / \mathrm{mL}$ OVA peptide (sequence 323-339; GenScript) for 72 hours. On day 3, Brefeldin 
A $(20 \mu \mathrm{g} / \mathrm{mL}$; eBioscience) was added to the cultures for 2 hours. At the end of the culture period, cells were incubated with antibodies against CD4 and the DO11.10 T-cell receptor (KJ-126) for 20 minutes on ice prior to fixation in $1 \%$ formalin solution for 20 minutes. Cells were incubated in permeabilization buffer (PBS $+0.1 \%$ saponin $+10 \%$ FCS) for 5 minutes at room temperature before the following anti-cytokine antibodies were added for 30 minutes: tumor necrosis factor (TNF) $\alpha$-eF450, interferon (IFN) $\gamma$-eF450, interleukin (IL)10-PE, IL5-PE, IL17-PE-Cy7, and IL4-APC (all eBioscience).

\section{Co-localization studies of PS particles with lysosomes}

For co-localization studies between lysosomes and PS particles, BMDCs were incubated in 8-well chamber slides (1 $\mu$-Slide ibiTreat microscopy chamber; ibidi $\mathrm{GmbH}$, Martinsried, Germany) with 20 or 1,000 nm PS particles with or without concomitant LPS for 16 hours. Following PS-particle exposure, BMDCs were washed with IMDM, and the plasma membrane stained with CellMask ${ }^{\mathrm{TM}}$ Orange (6 $\mu \mathrm{g} / \mathrm{mL}$, Molecular Probes) and lysosomes with LysoTracker ${ }^{\circledR}$ Deep Red ( $1 \mu \mathrm{M}$, Molecular Probes) during 1 hour at $37^{\circ} \mathrm{C}$. The optimal amount of LysoTracker ${ }^{\circledR}$ Deep Red was titrated prior to co-localization studies and adjusted to $1 \mu \mathrm{M}$ (data not shown). All micrographs were taken with a Zeiss LSM 710 (previously described). Micrographs used for three-dimensional reconstruction were taken with a $63 \times$ oil objective (previously described) and a digital zoom of $3 \times$, and image processing was performed using Imaris ${ }^{\circledR}$ software. Micrographs used to analyze co-localization were taken with a $40 \times$ oil objective (EC Plan-Neofluar $40 \times / 1.30$ Oil DIC M27/a $=0.21 \mathrm{~mm}$ ) and image analysis was performed using ImageJ (NIH, Bethesda, Maryland, USA) software. The degree of co-localization was analyzed in single cells (cropped from LSM images) by assessing the Pearson coefficient. This is an intensity-based analysis where linear correlation between 2 channels (here lysosomes and PS particles) is measured, giving a value between +1 and -1 , where 1 is total positive correlation, 0 is no correlation, and -1 is total negative correlation. Forty cells were analyzed for each condition.

\section{Assessment of lysosome permeability}

Lysosome permeability of BMDCs that were incubated for 16 hours with LPS and/or PS particles was measured by flow cytometry using the dye acridine orange (AO; Molecular Probes). BMDCs were stained at $37^{\circ} \mathrm{C}$ with $5 \mu \mathrm{g} / \mathrm{mL}$ AO for 15 minutes prior to flow cytometry analysis.

\section{ER stress measurement}

Total RNA was extracted from BMDCs treated with PS particles and/or LPS for 16 hours (NucleoSpin ${ }^{\circledR}$ RNA isolation kit; Macherey-Nagel, Oensingen, Switzerland). Complementary DNA was synthesized from $2 \mu \mathrm{g}$ of RNA using the Omniscript ${ }^{\mathrm{TM}}$ RT kit (Qiagen, Hombrechtikon, Switzerland). Real-time polymerase chain reactions (PCR) were performed using specific primers for GRP78 (5' AGCCCACCGTAACAATCAAG and 5' TCCAGCCATTCGATCTTTTC), CHOP (5' CTGCCTTTCACCTTGGAGAC and 5' CGTTTCCTGGGGATGAGATA), spliced XBP1 (5' GAGTCCGCAGCAGGTGCAG and 5' ACTGTGAGCGTCCAGCAGGC), IL-6 (5' GGTGGACAACCACGGCCTTCCC and 5'AAGCCTCCGACTTGTGAAGTGGT) and IL- $1 \beta$ (5' AAAGCCTCGTGCTGTCGGACC and 5' CAGGGTGGGTGTGCCGTCTT) (Microsynth, Balgach, Switzerland) and Fast SYBR Green Master Mix (Applied Biosystems ${ }^{\circledR}$, Lucerne, Switzerland). Reactions were performed using a 7500 Real-Time PCR System (Applied Biosystems ${ }^{\circledR}$ ). Gene expression was normalized to 18S RNA (5' CGCCGCTAGAGGTGAAATTCT and 5' CATTCTTGGCAAATGCTTTCG). Tunicamycin ( $2 \mu \mathrm{g} / \mathrm{mL}$; Sigma-Aldrich) was used as positive control. ${ }^{19}$

\section{Statistical analysis}

All data are presented as mean \pm SEM (standard error of the mean). Data were analyzed with ANOVA (one-way analysis of variance) followed by Bonferroni post hoc test. Data were analyzed using GraphPad Prism ${ }^{\circledR}$ (La Jolla, CA, USA) software. Values were considered significantly different with $P<0.05$ (*), $P<0.005$ (**), or $P<0.001$ (***).

\section{Results}

\section{PS-particle characterization}

Size of PS particles was measured with DLS in both PBS and cell medium containing 10\% FCS. We observed a larger size of $20 \mathrm{~nm}$ PS particles than the dimensions provided by the manufacturer $(31.3 \mathrm{~nm}$ in PBS, $43.9 \mathrm{~nm}$ in cell medium). When $20 \mathrm{~nm}$ PS particles were incubated in medium containing FCS, there was a modest increase in size compared with PBS, though this was still in the nanoscale. For 1,000 nm PS particles, results from DLS measurements confirmed the manufacturer specifications, both in PBS and cell medium (Table S1). Most importantly, for both particle sizes, DLS measurements and visual inspection excluded aggregation. Furthermore, samples were under observation for more than 3 days, during which period, stability was preserved. As previously published, the zeta potential for 
$20 \mathrm{~nm}$ PS particles was $-15.4 \pm 1.4 \mathrm{mV}$, and for $1,000 \mathrm{~nm}$ PS particles, $-17.5 \pm 1.9 \mathrm{mV} .{ }^{15}$

\section{PS particles do not induce apoptosis or cell death in BMDCs}

Prior to testing the immunomodulatory effects of PS particles on BMDC functional activity, we screened both PS-particle sizes for potential cytotoxic effects. BMDCs were exposed for 16 hours (overnight) to 20 or $1,000 \mathrm{~nm}$ PS particles at concentrations ranging from 5 to $100 \mu \mathrm{g} / \mathrm{mL}$ with or without concomitant LPS. Levels of apoptotic and dead cells were assessed by flow cytometry using Annexin $\mathrm{V}$ and propidium iodide stainings, respectively. We did not observe an increase in apoptosis or cell death in BMDCs treated with either size of PS particle compared with the untreated group (Figure 1). Additionally, BMDC morphology was unaltered by $25 \mu \mathrm{g} / \mathrm{mL}$ PS-particle or LPS treatment as demonstrated with light microscope pictures (Figure S1). For further experiments, a concentration of $25 \mu \mathrm{g} / \mathrm{mL}$ of PS particles was employed, as this concentration did not induce apparent cytotoxicity and has been shown in another study to be ideal in terms of fluorescence signal and cell viability. ${ }^{20}$

\section{PS-particle size determines internalization by BMDCs}

To determine the uptake of both sizes of PS particles by BMDCs at different stages of maturation, immature or LPS-matured BMDCs were treated with $25 \mu \mathrm{g} / \mathrm{mL} 20$ or $1,000 \mathrm{~nm}$ PS particles during a time course of 10, 20, 40, 60, and 90 minutes, and 2, 4, 8, and 16 hours. Cells were subsequently washed and labeled with antibodies to CD11c and $\mathrm{CD} 11 \mathrm{~b}$ and analyzed by flow cytometry. According to the literature, myeloid or conventional DCs that are in vitro derived from bone-marrow cells in the presence of GM-CSF consistently show stable high expression of CD11c and CD11b. ${ }^{16,21}$ Frequencies of CD $11 c^{+} / C D 11 b^{+}$BMDCs containing PS particles were then determined (Figure 2A). Our results showed significantly higher uptake of $20 \mathrm{~nm}$ compared with 1,000 nm PS particles in non-LPS-exposed BMDCs at all time-points studied (Figure 2B, left). Uptake of $20 \mathrm{~nm}$ PS particles reached a plateau after 20 minutes incubation, with approximately $75 \%$ of BMDCs positive for PS particles. In contrast, LPS-exposed BMDCs showed similar uptake for both particle sizes at early time-points, but at later time-points a significantly higher uptake of $20 \mathrm{~nm}$ PS particles compared with 1,000 nm PS particles occurred (Figure 2B, right). We observed that uptake of $20 \mathrm{~nm}$ PS particles by BMDCs remained stable during all time-points studied, irrespective of LPS exposure, whereas uptake of $1,000 \mathrm{~nm}$ PS particles at early timepoints (10-90 minutes) was significantly increased in LPS-exposed BMDCs compared with non-LPS-exposed BMDCs (Figure 2B).

To specifically determine whether PS particles were localized intra- or extracellular, we performed an in-depth analysis of BMDCs by LSM (Figure 2C). As early as 10 minutes after PS-particle exposure, high numbers of $20 \mathrm{~nm}$ PS particles were detected inside BMDCs (Figure 2C [B and G]), compared with low numbers of $1,000 \mathrm{~nm}$ PS particles (Figure 2C [C and H]). In particular, lower magnification analyses provided evidence that although the majority of BMDCs contained $20 \mathrm{~nm}$ PS particles at early time-points, only a small number of BMDCs had taken up 1,000 nm PS particles (data not shown). Moreover, most $20 \mathrm{~nm}$ PS particles were localized intracellular, whereas a proportion of $1,000 \mathrm{~nm}$ PS particles were detected on the outer cell surface. Importantly, no PS particles were localized in cell nuclei at any time-point.
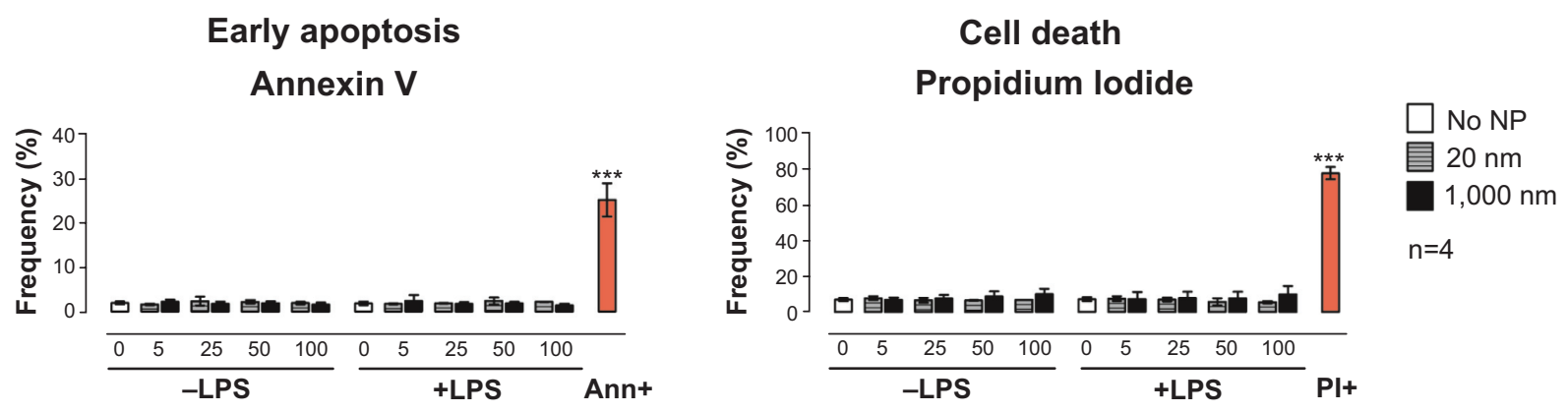

Figure I Assessment of BMDC viability using flow cytometry.

Notes: Frequencies of BMDCs in early apoptosis phase (left) or dead (right) following I6-hour incubation with $20 \mathrm{~nm}$ (gray) or I,000 nm (black) PS particles compared with non-treated cells (white), stained for Annexin $\mathrm{V}$ and PI, and measured by flow cytometry. Concentrations of PS particles ranged from 5 to I00 $\mu \mathrm{g} / \mathrm{mL}$. Ann+ (positive control for apoptosis) was obtained by fixation of BMDCs with $1 \%$ formalin for 40 minutes. $\mathrm{PI}+$ (positive control for cell death) was obtained by placing $\mathrm{BMDCs}$ for 30 minutes at $-80^{\circ} \mathrm{C}$. Bar graphs show mean $\pm \mathrm{SEM}$; ***P $<0.00$ I (positive control versus no NP); $n=4$.

Abbreviations: Ann, annexin; BMDC, bone marrow-derived dendritic cell; LPS, lipopolysaccharide; NP, nanoparticle; PI, propidium iodide; PS, polystyrene; SEM, standard error of the mean. 
A

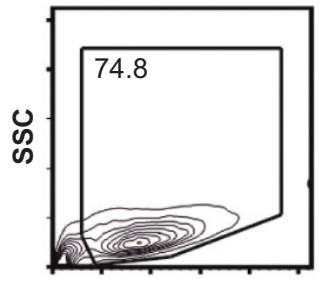

FSC

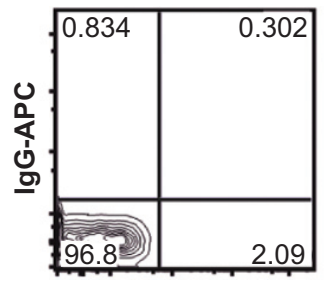

IgG-APC-Cy7

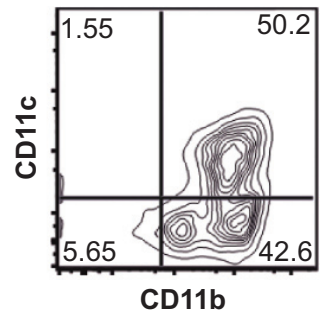

B

Particle uptake -LPS

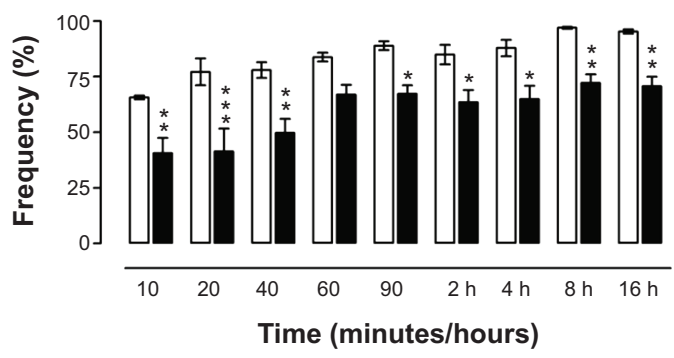

Particle uptake +LPS

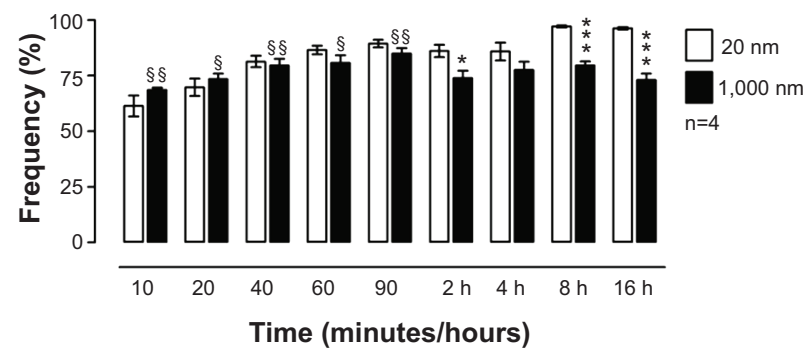

C 10 minutes incubation

90 minutes incubation
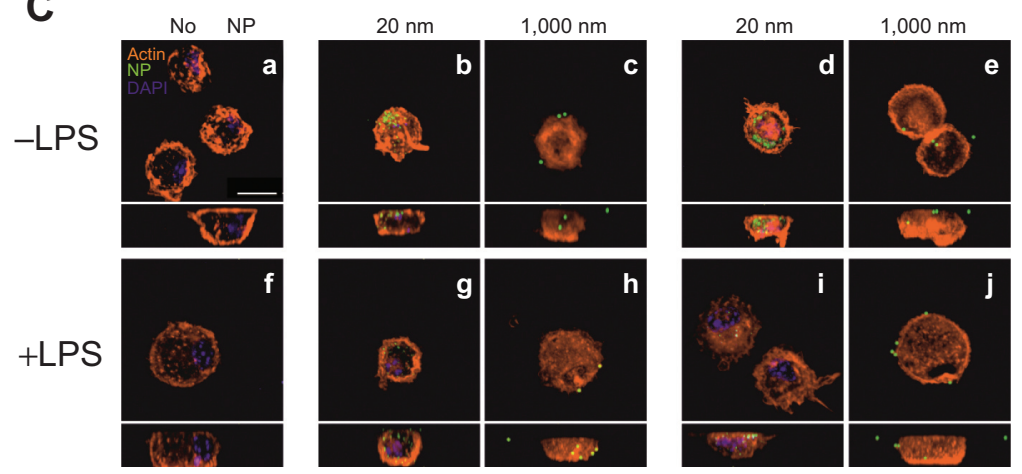

Figure 2 Uptake of PS particles by BMDCs determined by flow cytometry and LSM.

Notes: (A) Flow cytometry analysis included gating on $\mathrm{CDI} \mathrm{lb} \mathrm{b}^{+} / \mathrm{CDI}$ l c $\mathrm{c}^{+} \mathrm{BMDCs}$ based on their relevant isotype controls. (B) Frequencies of positive cells for $20 \mathrm{~nm}$ (white) or I,000 nm (black) PS particles were subsequently determined. Left panel shows data for immature BMDCs (-LPS), while right panel shows data for mature BMDCs (+LPS). White bars, $20 \mathrm{~nm}$; black bars, I,000 nm. Bar graphs show mean \pm SEM; $* P<0.05, * * P<0.005, * * * P<0.00$ I $(20 \mathrm{~nm}$ versus I,000 nm PS particles); $\$ P<0.05, \S \S P<0.005(-$ LPS versus +LPS); $n=4$. (C) Confirmation of PS-particle uptake by BMDCs determined by LSM. Upper micrographs (a-e) show uptake by immature BMDCs (-LPS), while lower micrographs $(f-j)$ show uptake by mature BMDCs (+LPS). xy-projections (top panels) and xz-projections (lower panels) were obtained from three-dimensional stacks of consecutive optical sections. Orange, actin; green, particles; blue, DAPI (nucleus). White bar: $10 \mu \mathrm{m}$. Magnification: $63 \times+3 \times$ digital zoom. One representative experiment from four independent experiments is shown.

Abbreviations: BMDC, bone marrow-derived dendritic cell; DAPI, 4',6-diamidino-2-phenylindole; LPS, lipopolysaccharide; LSM, laser scanning microscopy; NP, nanoparticle; PS, polystyrene; SEM, standard error of the mean.

In summary, flow cytometry and LSM analyses demonstrated that both 20 and 1,000 nm PS particles were successfully taken up by BMDCs in a time-dependent manner, although $20 \mathrm{~nm}$ PS particles were internalized more rapidly and to a greater extent compared with 1,000 nm PS particles.

\section{Stable BMDC phenotype following treatment with PS particles}

We next aimed to analyze possible changes in BMDC phenotype and expression of co-stimulatory molecules following PS-particle exposure with or without concomitant LPS treatment. Employing flow cytometry with relevant isotype controls, we gated $\mathrm{CD} 11 \mathrm{c}^{+} / \mathrm{CD} 11 \mathrm{~b}^{+} \mathrm{BMDCs}$ to determine $\mathrm{MHC}$ class $\mathrm{II}^{+}\left(\mathrm{MHCII}^{+}\right), \mathrm{CD} 40^{+}$, and $\mathrm{CD} 86^{+}$ cell frequencies (Figure 3A). The frequencies of $\mathrm{CD} 40^{+}$and $\mathrm{CD} 86^{+}$cells significantly increased upon LPS exposure, but were unaffected by PS-particle treatment of both sizes. In contrast, frequencies of $\mathrm{MHCII}^{+}$cells remained unchanged by both LPS and/or PS-particle treatment (Figure 3B). Taken together, these findings suggest that neither 20 nor $1,000 \mathrm{~nm}$ PS particles induced alterations in BMDC surface phenotype 


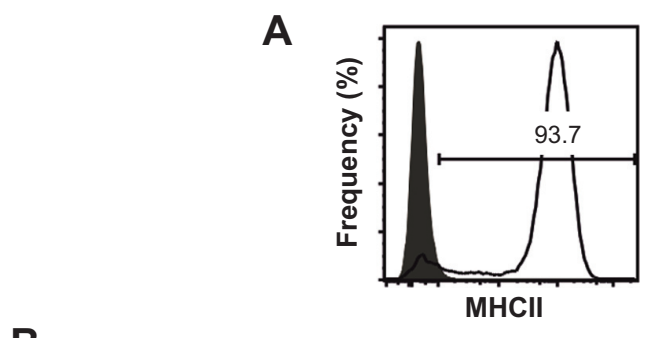

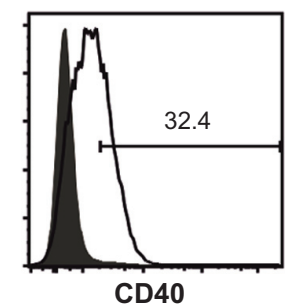

CD11c

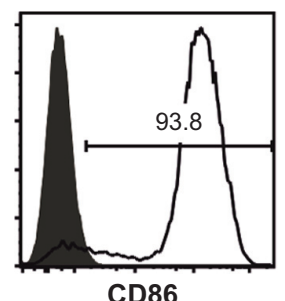

CD86
B
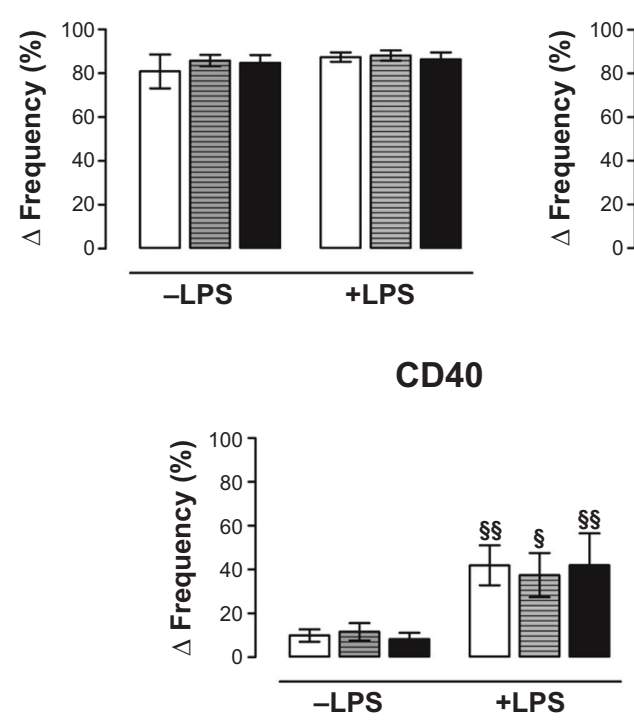

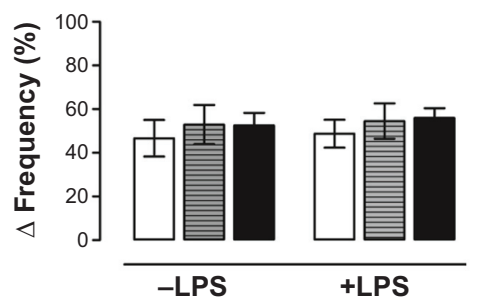

CD86

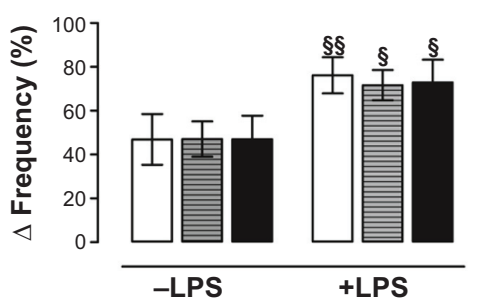

MHCII

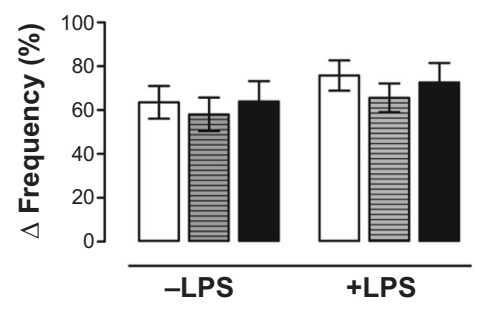

Figure 3 Expression of phenotype and co-stimulatory markers utilizing flow cytometry.

Notes: $(\mathbf{A}) \mathrm{CDI} \mathrm{lb}^{+} / \mathrm{CD} / \mathrm{Ic}^{+} \mathrm{BMDCs}$ were gated based on their relevant isotype controls. Frequencies of expression of MHCIl, CD40, and CD86 (open histograms) were subsequently determined based on their adequate isotype control (solid histograms). (B) Frequencies of BMDCs positive for the phenotypic markers CDIIb, CDIIc, and MHCII (upper panels) and for the co-stimulatory molecules CD40 and CD86 (lower panels) were analyzed. Results are expressed as $\triangle$ frequency $=$ measured sample frequency minus measured isotype control frequency. White bars, no particles; gray bars, $20 \mathrm{~nm}$; black bars, I,000 nm PS particles. Bar graphs show mean \pm SEM; $\S P<0.05$, $\S \S P<0.005$ (-LPS versus +LPS); $\mathrm{n}=4$ (CD40 and CD86), $\mathrm{n}=8$ (CDI I b, CDI Ic, and MHCII).

Abbreviations: BMDC, bone marrow-derived dendritic cell; LPS, lipopolysaccharide; NP, nanoparticle; PS, polystyrene; SEM, standard error of the mean.

and activation in the absence or presence of LPS-dependent maturation.

\section{Size $20 \mathrm{~nm}$ PS particles modulate antigen degradation in BMDCs}

To investigate potential particle-mediated alterations of BMDC function, we investigated the capacity of BMDCs to take up and degrade antigen following PS-particle treatment. As previously reported, OVA coupled to Alexa647 and BSA coupled to DQ-Red were used to analyze antigen uptake and antigen degradation, respectively..$^{18,22-24}$ The flow cytometry gating strategy from one representative experiment for OVA uptake and $\mathrm{BSA}$ degradation by $\mathrm{CD} 11 \mathrm{c}^{+} / \mathrm{CD} 11 \mathrm{~b}^{+} \mathrm{BMDCs}$ is shown in Figure 4A. In the absence of concomitant LPS exposure, OVA uptake was similar in $\mathrm{CD} 11 \mathrm{c}^{+} / \mathrm{CD} 11 \mathrm{~b}^{+}$ BMDC cultures without particles compared with those incubated with 20 or 1,000 nm PS particles. However, antigen uptake was reduced in LPS-treated BMDCs compared with
non-LPS-treated BMDCs, independently of PS-particle exposure (Figure 4B, upper panels). Decreased antigen degradation occurred in BMDCs treated with $20 \mathrm{~nm}$ PS particles but not with 1,000 nm PS particles, when compared with the untreated group, both in the presence or absence of LPS (Figure 4B, lower panels). Therefore, although PS particles did not alter antigen uptake by BMDCs, we observed decreased antigen degradation following exposure to $20 \mathrm{~nm}$ PS particles, both in immature (-LPS) and mature (+LPS) BMDCs.

\section{Antigen-specific T-cell stimulation by BMDCs remains stable following treatment with PS particles}

We employed an in vitro OVA-specific DO11.10 ${ }^{+} \mathrm{CD}^{+}$ $\mathrm{T}$-cell proliferation assay to clarify whether alterations in antigen degradation induced by PS-particle treatment affect downstream antigen presentation and activation of $\mathrm{CD} 4^{+}$ T-cells by BMDCs. Following treatment with PS particles 
A

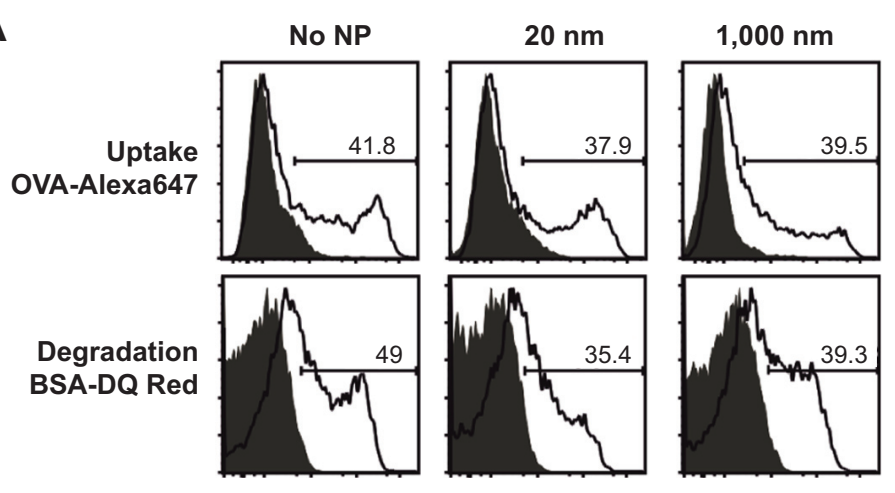

B
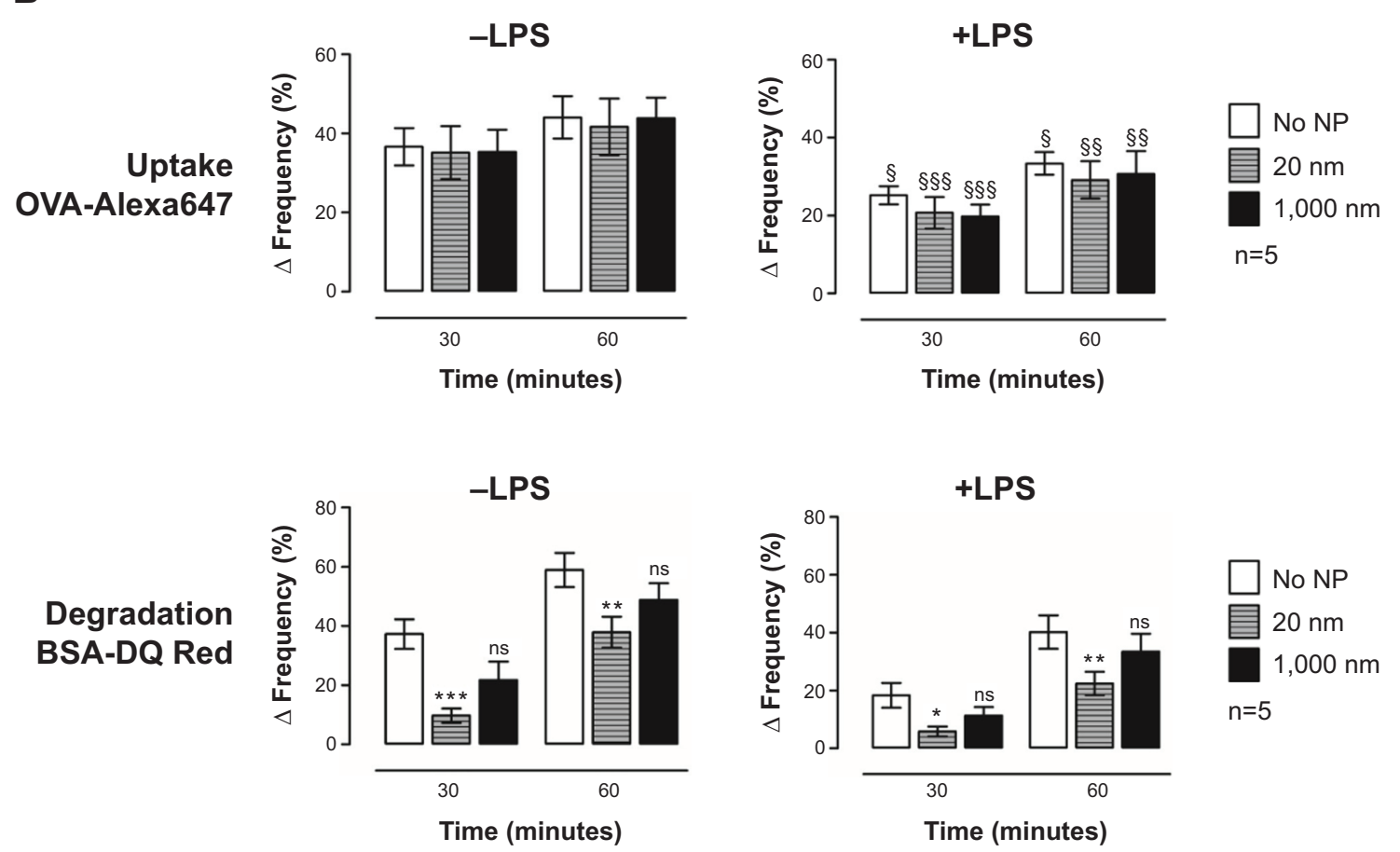

Figure 4 Antigen uptake and antigen degradation in the presence of particles and/or LPS, measured utilizing flow cytometry.

Notes: Cells were incubated with OVA-Alexa647 (antigen uptake) or BSA-DQ Red (antigen degradation) during a time course of $0-60$ minutes at $37^{\circ} \mathrm{C}$ or on ice (controls). (A) Histogram plots of one representative experiment. Negative controls (solid histograms) were obtained with cells kept on ice. Open histograms represent antigen uptake (upper panel) or antigen degradation (lower panel) after 60 minutes. (B) Bar graphs show capacity of BMDCs to take up (upper panel) and degrade (lower panel) antigen after 16 hours incubation with $20 \mathrm{~nm}$ (gray bars) or $1,000 \mathrm{~nm}$ (black bars) PS particles compared with non-treated cells (white bars). Results are expressed as $\Delta$ frequency = measured sample frequency (at time-point 30 or 60 minutes) minus measured control frequency (at time-point 0 minutes). Bars show mean $\pm S E M ; * P<0.05$, $* * P<0.005$, $* * * P<0.001$ (no NP versus $20 \mathrm{~nm}$ ); $\S P<0.05, \S \S P<0.005, \S \S \S P<0.00$ I (-LPS versus + LPS), ns, non-significant; $n=5$.

Abbreviations: BMDC, bone marrow-derived dendritic cell; BSA, bovine serum albumin; LPS, lipopolysaccharide; NP, nanoparticle; OVA, ovalbumin; PS, polystyrene; SEM, standard error of the mean.

with or without LPS, BMDCs were co-cultured with CFSElabeled transgenic $\mathrm{CD} 4^{+} \mathrm{T}$-cells specific for an $\mathrm{H}-2^{\mathrm{d}}$-restricted OVA peptide. DO11.10+ $\left(\mathrm{KJ}-126^{+}\right) \mathrm{CD} 4^{+} \mathrm{T}$-cells were gated (Figure 5A), and responses to OVA peptide and whole OVA protein were assessed by analyzing T-cell proliferation (CFSE dilutional profiles) after 72 hours of culture. The expansion index (EI) was measured based on CFSE profiles with the Flow Jo software. The EI is the ratio of final cell count to starting cell count and therefore represents the fold expansion during culture. ${ }^{25}$ Independently of LPS, the EI remained unchanged for cells treated with PS particles as compared with untreated cells both for OVA peptide and OVA protein pulsed BMDCs (Figure 5B and C). Significant increases in $\mathrm{CD}^{+} \mathrm{T}$-cell proliferation occurred in LPS-exposed BMDCs as compared with non-LPS-exposed cultures (Figure 5C). These data therefore suggest that antigen-specific T-cell stimulation by antigen-pulsed BMDCs, using either OVA peptide (antigen-degradation independent) and whole OVA protein (antigen-degradation dependent), remained unaffected by PS-particle exposure of either size. 
A

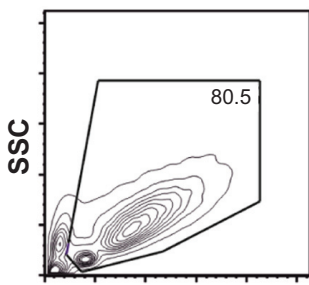

FSC

B

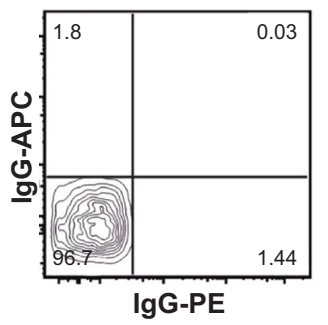

C

\section{Expansion index -LPS}

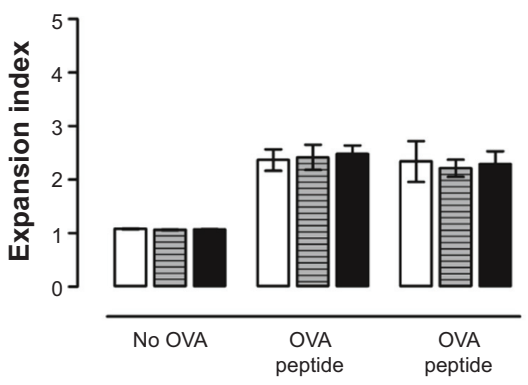

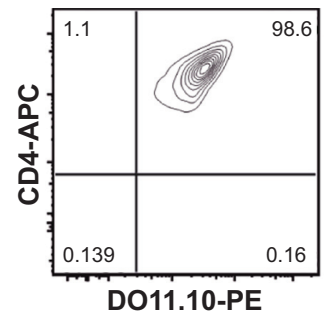

Expansion index + LPS

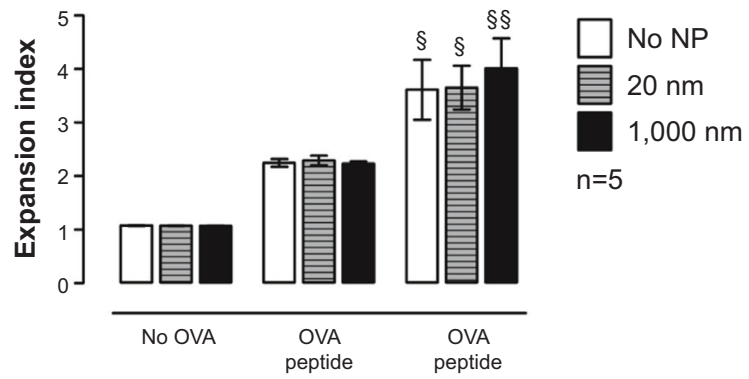

Figure 5 In vitro OVA-specific DOII.10+/CD4 ${ }^{+}$T-cell stimulatory capacity of immature and mature BMDCs incubated with $25 \mu \mathrm{g} / \mathrm{mL}$ of 20 or $1,000 \mathrm{~nm}$ PS particles in the presence or absence of OVA peptide or OVA protein.

Notes: (A) Gating strategy consists of an FSC/SSC gate (left panel) and an isotype-control gate for CD4+/DOII.I0+ T-cells (middle and right panels). Proliferation of DOII.1 $0^{+} / \mathrm{CD}^{+} \mathrm{T}$-cells was assessed with the help of Flow Jo software by calculating the expansion index. Results show proliferation of T-cells following co-culture with non-LPS-exposed (B) and LPS-exposed (C) BMDCs in absence of OVA, with $20 \mu \mathrm{g} / \mathrm{mL}$ OVA peptide or with $20 \mu \mathrm{g} / \mathrm{mL}$ whole OVA protein. White bars, no particles; gray bars, $20 \mathrm{~nm}$; black bars, I,000 nm PS particles. Bars show mean \pm SEM; $\S P<0.05, \S \S P<0.005$ (-LPS versus + LPS); $n=5$.

Abbreviations: BMDC, bone marrow-derived dendritic cell; LPS, lipopolysaccharide; NP, nanoparticle; OVA, ovalbumin; PS, polystyrene; SEM, standard error of the mean.

\section{PS particle-treated BMDCs do not alter antigen-specific CD4 ${ }^{+}$T-cell cytokine profiles}

Although PS-particle exposure did not alter BMDC antigen presentation and $\mathrm{CD} 4^{+} \mathrm{T}$-cell proliferation, we next determined whether immune responses may be affected by alteration of T-cell cytokine profiles. To test this, DO11.10 $/ \mathrm{CD}^{+}$ T-cells were co-cultured with PS particle-exposed BMDCs ( \pm LPS) for 72 hours in the presence or absence of $20 \mu \mathrm{g} / \mathrm{mL}$ OVA peptide. Production of the cytokines IFN- $\gamma$ and TNF- $\alpha$ (Th-1), IL-4 and IL-5 (Th-2), IL-17 (Th-17), IL-10 (regulatory T-cells $)^{26}$ by $\mathrm{CD}^{+} \mathrm{T}$-cells was assessed by intracellular staining and flow cytometry (Figure 6 ). OVA peptide \pm LPS, but not LPS alone, induced cytokine production that was not affected by PS-particle treatment of BMDCs.

\section{Size $20 \mathrm{~nm}$ PS particles co-localize with lysosomes without increasing lysosome permeability}

To understand why antigen degradation was decreased following treatment with $20 \mathrm{~nm}$ PS particles but not with larger 1,000 nm PS particles, we analyzed the extent to which
PS particles co-localized with lysosomes, as these organelles are the site where antigen degradation takes place. Following 16 hours incubation with $25 \mu \mathrm{g} / \mathrm{mL}$ PS particles, lysosomes were stained using the lysosomal marker LysoTracker ${ }^{\circledR}$ Deep Red, which labels acidic organelles, ${ }^{3,27-29}$ and examined by confocal microscopy. While almost all $20 \mathrm{~nm}$ PS particles co-localized with lysosomes, fewer 1,000 nm PS particles co-localized in lysosomes (Figure 7A, arrows). We next quantified co-localization using the Pearson coefficient. A significant increase in co-localization with lysosomes occurred following incubation with $20 \mathrm{~nm}$ compared with $1,000 \mathrm{~nm}$ PS particles in both untreated and LPS-treated BMDCs (Figure 7B). In addition, co-localization of $20 \mathrm{~nm}$ PS particles in LPS-treated BMDCs was higher than in non-treated BMDCs (Figure 7B). To exclude that PS-particle fluorescence changes occurred at acidic $\mathrm{pH}$ in lysosomes, potentially influencing particle detection, we investigated the emission fluorescence intensity of PS particles in buffer solutions at $\mathrm{pH} 5$ and $\mathrm{pH} 7$ at different time-points. No significant differences in fluorescence intensity occurred at $\mathrm{pH} 5$ and $\mathrm{pH} 7$ for both 20 and 1,000 nm PS particles at all time-points (Figure S2).

We further assessed whether PS particles could affect lysosomal membrane integrity and therefore induce leakage 

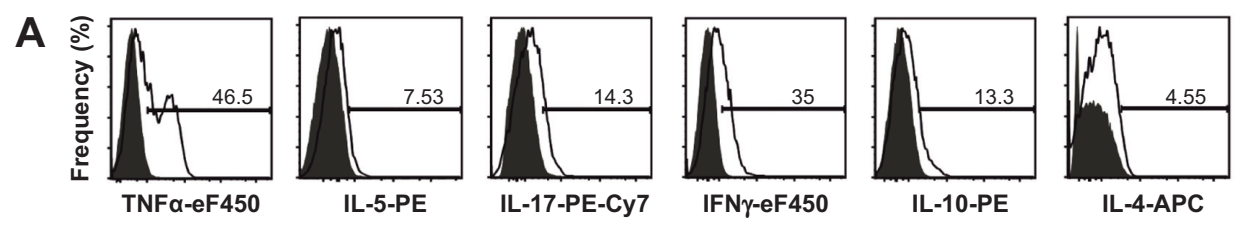

B

IFN- $\gamma$

TFN- $\alpha$

IL-17

Th-1
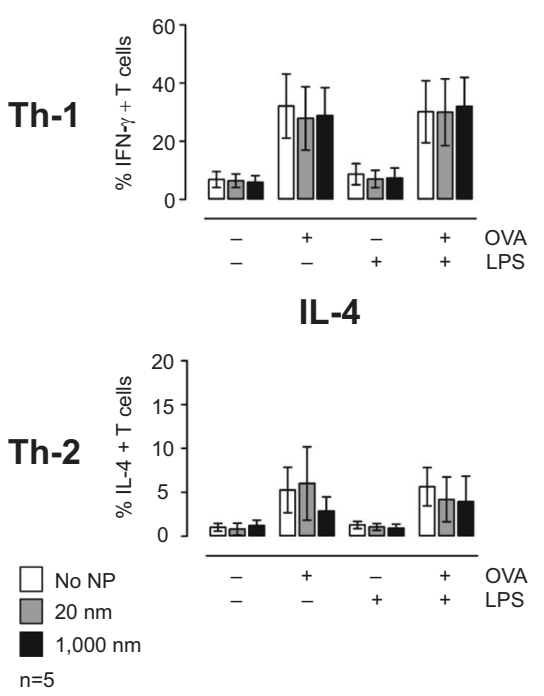

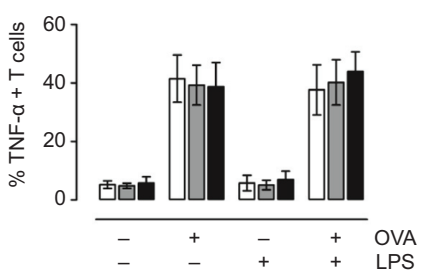

IL-5

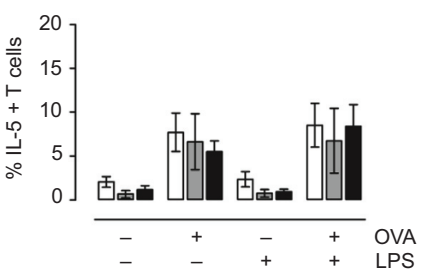

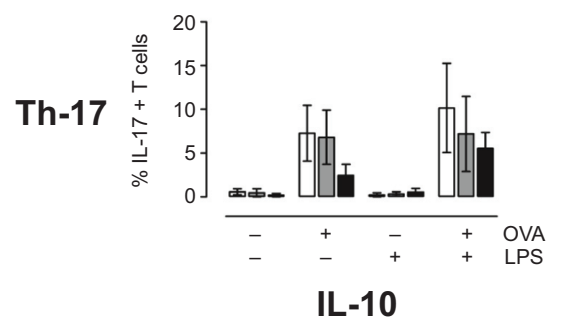

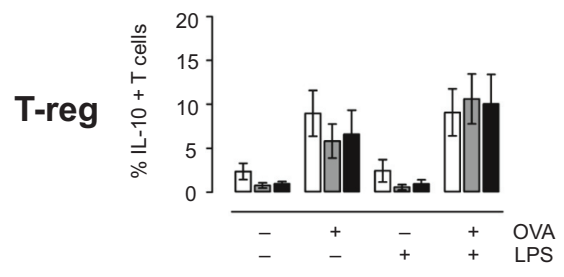

Figure 6 Cytokine production by DOII.10 transgenic CD4+ T-cells following 72 hours co-culture with BMDCs in absence or presence of LPS and OVA, analyzed by flow cytometry.

Notes: (A) Gating strategy consists in an isotype-control gate for CD4+/DOII.10+ T-cells. Frequencies of positive T-cells for different cytokines (open histograms) were determined based on respective isotype controls (solid histograms). (B) Frequencies of CD4 $4^{+}$DOII.I $0^{+}$T-cells positive for Th-I cytokines IFN- $\gamma$ and TNF- $\alpha$, Th- 2 cytokines IL-4 and IL-5, Th- 17 cytokine IL- I7, and T-reg cytokine IL- 10 are shown. Results are expressed as $\Delta$ frequency = measured sample frequency minus measured isotype control frequency. White bars, no particles; gray bars, $20 \mathrm{~nm}$; black bars, I,000 nm PS particles. Bars show mean \pm SEM; $\mathrm{n}=5$.

Abbreviations: BMDC, bone marrow-derived dendritic cell; IFN, interferon; IL, interleukin; LPS, lipopolysaccharide; NP, nanoparticle; OVA, ovalbumin; PS, polystyrene; SEM, standard error of the mean; Th, T helper; TNF, tumor necrosis factor; T-reg, regulatory T-cells.

of lysosomal enzymes into the cytosol, by using the dye AO. AO accumulates in lysosomes and leads to red fluorescence, which decreases when the dye leaks out of the lysosomes into the cytosol due to increased permeability. AO-negative gating was used to assess the percentage of $\mathrm{AO}^{\text {low }} \mathrm{BMDCs}$, which represents cells with lower AO fluorescence intensity, thus permeable lysosomes (Figure 7C). We observed that incubation with $25 \mu \mathrm{g} / \mathrm{mL}$ of both 20 and 1,000 nm PS particles did not increase permeability of lysosomes (Figure 7D), providing evidence that lysosomes remain intact at the concentrations utilized.

\section{PS particles do not induce ER stress in BMDCs}

One stage in antigen degradation involves synthesis of $\mathrm{MHC}$ class II molecules in the ER. Following synthesis, MCHII molecules are transported to endosomal vesicles, where they are loaded with antigen-derived peptides. ${ }^{30}$ To identify potential adverse effects of PS particles on this phase of antigen processing, we assessed ER stress in BMDCs which were previously incubated with $25 \mu \mathrm{g} / \mathrm{mL}$ PS particles.
The ER stress response is triggered when an accumulation of misfolded or unfolded proteins is found in the lumen of the ER under stress conditions. ${ }^{31,32}$ Expression of different ER stress gene markers such as GRP78 chaperone and adaptive pathways CHOP and spliced XBP1 were measured by realtime-PCR. Expression of the pro-inflammatory cytokines IL-1 $\beta$ and IL-6 was also determined. Tunicamycin, a known ER stress inducer, was employed as positive control for ER stress. Treatment of BMDCs with PS particles did not trigger an increased expression of messenger RNA (mRNA) encoding GRP78, CHOP and spliced XBP1, or transcription of the genes encoding the cytokines IL- $1 \beta$ and IL-6 (Figure 8 ). Treatment of cells with LPS induced, as expected, an expression of IL-1 $\beta$ mRNA; this was, however, unchanged in the presence of PS particles. These results indicate that PS particles did not induce an apparent ER stress response in BMDCs.

\section{Discussion}

Despite recent intensive research in the field of nanomedicine, fundamental knowledge about nanomaterial-cell interactions 
A

A
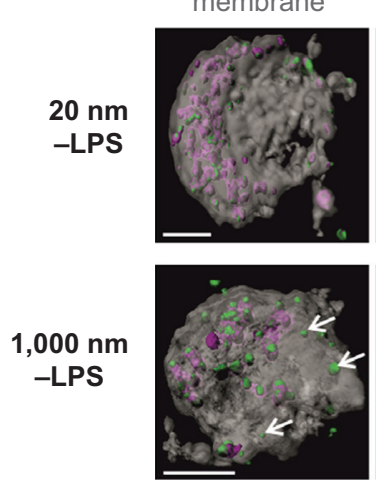

C

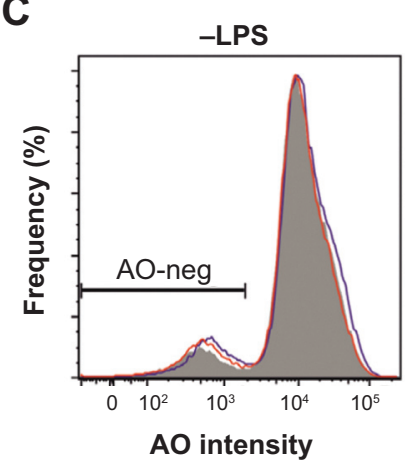

Lysosomes

PS particles
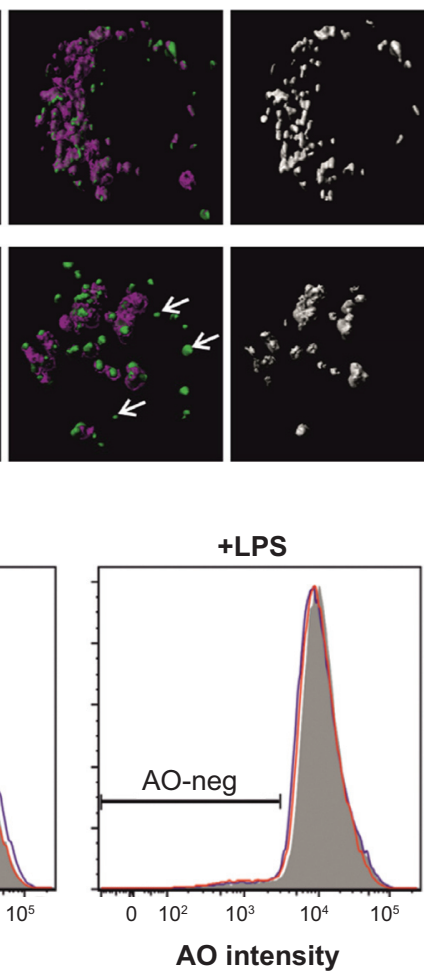

Co-localization Lyso/PS
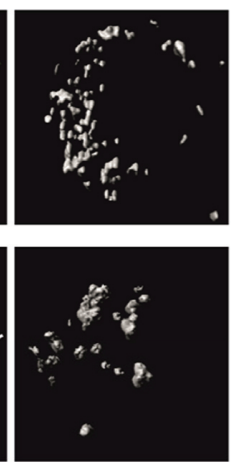

D

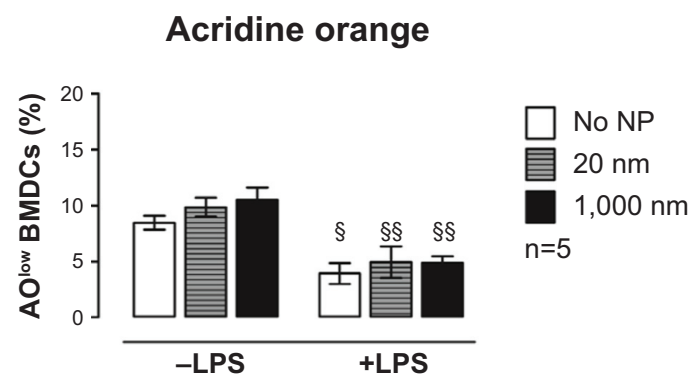

Figure 7 Interaction of PS particles with lysosomes analyzed by LSM and flow cytometry.

Notes: (A) Upper micrographs show co-localization with $20 \mathrm{~nm}$ PS particles in immature BMDCs (-LPS), whereas lower micrographs show co-localization with I,000 nm PS particles. Micrographs were obtained from three-dimensional stacks of consecutive optical sections and analyzed with Imaris ${ }^{\circledR}$ software. Gray, cell membrane; green, PS particles; violet, lysosomes; white, co-localization of lysosomes and PS particles. White bars: $10 \mu \mathrm{m}$. Magnification: $63 \times+3 \times$ digital zoom. Arrows: PS particles not co-localized with lysosomes. One representative experiment from four independent experiments is shown. (B) Quantitative analysis of co-localization between PS particles and lysosomes using Image J software. Four independent experiments were performed, and for each condition, 40 cells in total were selected randomly and analyzed. White bars, -LPS; gray bars, +LPS. Bar graphs show mean \pm SEM; $* * P<0.005$, ***P $<0.001$. (C) Histogram plots for BMDCs stained with AO and analyzed by flow cytometry. AO-negative gating was used to assess the percentage of $A O^{\text {low }}$ BMDCs showing lysosomal leakage. No NP, gray solid histogram; $20 \mathrm{~nm}$, blue open histogram; I,000 nm, red open histogram. (D) Frequencies of $A O^{\text {low }} B M D C$ s following 16 hours incubation with $25 \mu \mathrm{g} / \mathrm{mL} 20 \mathrm{~nm}$ (gray) or I,000 nm (black) PS particles. Bars show mean $\pm S E M$; $\$ P<0.05, \S \S P<0.005$ (-LPS versus +LPS); $\mathrm{n}=5$.

Abbreviations: AO, acridine orange; BMDC, bone marrow-derived dendritic cell; LPS, lipopolysaccharide; LSM, laser scanning microscopy; NP, nanoparticle; PS, polystyrene; SEM, standard error of the mean.

is still insufficient, particularly in understanding effects on the immune system. It has been recognized to date that NP-cell interactions are affected by a number of diverse particle properties, such as size, shape, and surface charge. In this study, we aimed to analyze size-dependent effects on BMDCs during treatment with nanosized $(20 \mathrm{~nm})$ and microsized $(1,000 \mathrm{~nm})$ PS particles in an in vitro model. The scope of the present study was to focus on particle size in order to systematically and thoroughly analyze size-dependent effects, and therefore only PS particles were included in the present study. Further evaluations are in progress employing a recently established fluorescence-encoded gold-NP library with different surface modifications ${ }^{33}$ in order to understand surface-dependent effects. The underlying study is complementary to our recent work showing that in vivo lung DC subpopulations preferentially capture and traffic $20 \mathrm{~nm}$ PS particles compared with 1,000 nm PS particles, where smaller particles induce enhanced $\mathrm{CD} 4^{+} \mathrm{T}$-cell proliferation in draining lymph nodes..$^{15} \mathrm{In}$ the current study, we provided an exhaustive investigation into particle-dependent modulation of DC function and showed that treatment with particles did not affect cell viability, surface phenotype (CD11c and CD11b), or activation (CD40, CD86, and MHCII) markers. Although particle treatment did not modulate antigen uptake, $20 \mathrm{~nm}$ PS particles decreased the capacity of BMDCs to degrade antigen irrespective of LPS exposure, compared with larger 1,000 nm PS particles that did not modulate BMDC function. For both PS-particle sizes, BMDC-mediated antigen-specific stimulation of $\mathrm{CD} 4^{+} \mathrm{T}$-cells and induction of cytokines remained similar to non-particle-exposed control cells. Furthermore, we observed that co-localization of $20 \mathrm{~nm}$ PS particles with lysosomes was significantly higher compared with 1,000 nm PS particles, without measurable perturbation of lysosomal function or ER stress. 
GRP78

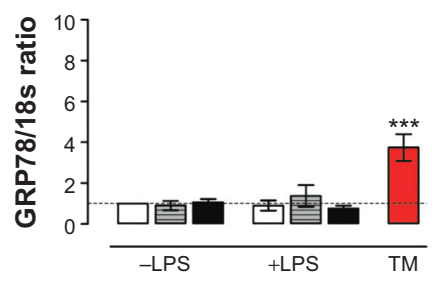

IL-6

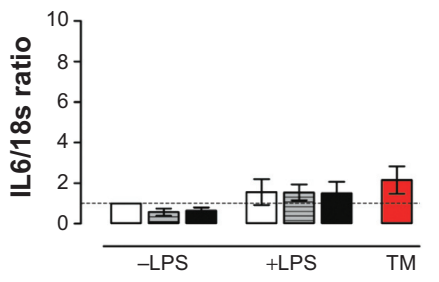

CHOP

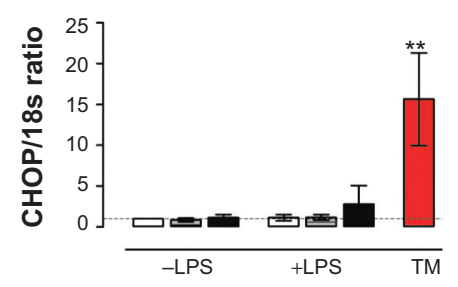

IL-1 $\beta$

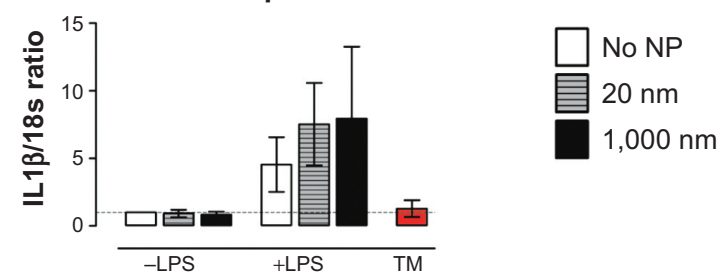

Figure 8 Messenger RNA expression level of different markers for endoplasmic reticulum stress measured by real-time-PCR in BMDCs following PS-particle exposure. Notes: Expression of GRP78, CHOP, and XBPI, as well as pro-inflammatory cytokines ILI- $\beta$ and IL-6 were assessed in cells left untreated (white) or incubated overnight with $25 \mu \mathrm{g} / \mathrm{mL} 20 \mathrm{~nm}$ (gray) or I,000 nm (black) PS particles. Expression levels of genes of interest were normalized to I8S gene expression. All ratios were normalized to the ratio of No NP - LPS (BMDCs unexposed to PS particles and untreated with LPS). Red bars show expression level for TM, a positive control for endoplasmic reticulum stress. Bar graphs show mean \pm SEM; $* * P<0.005$, *** $P<0.00$ I (TM versus no NP - LPS); $n=4$.

Abbreviations: BMDC, bone marrow-derived dendritic cell; LPS, lipopolysaccharide; NP, nanoparticle; PCR, polymerase chain reaction; PS, polystyrene; TM, tunicamycin; SEM, standard error of the mean; IL, interleukin.

We adopted a widely employed robust model utilizing submersed cultures of BMDCs to allow straightforward administration of PS particles and analyses by flow cytometry and confocal microscopy. Nevertheless, it is central to interpret the data in the context of the particle application method employed in this study (as described in the Methods section): for every particle size, a suspension containing $25 \mu \mathrm{g} / \mathrm{mL}$ of PS particles was administrated; hence, there is a 125,000 -fold difference in particle numbers at the extremes between 20 and 1,000 nm PS particles. This difference in particle number could theoretically influence particle uptake by BMDCs measured by determining fluorescence intensities. This potential bias in particle numbers is countered by limitations in detection sensitivity by flow cytometry: While fluorescence emitted from a single $1,000 \mathrm{~nm}$ PS particle is highly above detection threshold and can therefore easily be detected by flow cytometry, this is not the case for single $20 \mathrm{~nm}$ PS particles (data not shown). Therefore, with our approach that heavily relied on detecting and tracing sufficiently fluorescent particles over a wide size range, utilizing equal particle mass was the most appropriate strategy to account for size-dependent variations in fluorescence intensities. ${ }^{15}$ The resolution of microscopy is limited to approximately $200 \mathrm{~nm}$ in lateral and $500 \mathrm{~nm}$ in axial dimension. ${ }^{34}$ Although single $20 \mathrm{~nm}$ PS particles cannot be distinguished, the number of pixels generating a signal event can be quantified. As previously reported, we employed this approach to analyze both 20 and $1,000 \mathrm{~nm}$ PS particles ${ }^{15}$ or fluorescent-magnetic hybrid NPs. ${ }^{34}$

In line with our findings, a previous study by Frick et $\mathrm{l}^{35}$ showed that human DCs incubated with negatively charged phosphonated and sulfonated PS particles of an average diameter of 245 and $227 \mathrm{~nm}$, respectively, at concentrations of 25,75 , and $150 \mu \mathrm{g} / \mathrm{mL}$ for 24 hours does not cause significant apoptosis. Another study by Zupke et al ${ }^{20}$ determined that only larger PS particles (100 and $500 \mathrm{~nm}$ ) at a concentration of $50 \mu \mathrm{g} / \mathrm{mL}$ for 24,48 , and 72 hours induced significant cytotoxicity in NIH $3 \mathrm{~T} 3$ fibroblast cells compared with smaller PS particles (50 nm). In fact, even though PS particles employed in our study are often considered to be chemically inert and safe, it is essential to realize that such particles may induce biological effects partly associated with surface charge. ${ }^{36}$ It is indeed often reported that positively charged PS particles trigger cell death even at low concentrations when compared with neutral or negatively charged particles, indicating that the surface coating plays a major role in cell death induced by particles. ${ }^{3,37,38}$ Since we employed negatively charged PS particles for our exposures, the low levels of cell death found following PS-particle treatment can be explained by the negative surface charge of our PS particles. 
We also provided evidence for a differential uptake of 20 and 1,000 nm PS particles by immature (non-LPSexposed) BMDCs. In immature BMDCs, uptake of $20 \mathrm{~nm}$ PS particles reached an early plateau after 20 minutes, whereas this only occurred after 60 minutes for $1,000 \mathrm{~nm}$ PS particles. The frequency of particle-positive BMDCs was always lower for $1,000 \mathrm{~nm}$ than for $20 \mathrm{~nm}$ PS particles. Employing cell lines, a human in vitro study with negatively charged PS particles ranging from $40 \mathrm{~nm}$ to $2 \mu \mathrm{m}$ showed that uptake was also size-dependent for all cell lines tested. ${ }^{39} \mathrm{As}$ expected, it was also demonstrated that phagocytic cells have a much higher internalization capacity compared with other cell types, although all NPs tested still could enter all cell lines. ${ }^{39}$ Moreover, when monitoring size-dependent uptake, Johnston et al ${ }^{40}$ reported limited uptake of $200 \mathrm{~nm}$ compared with $20 \mathrm{~nm}$ PS particles in human hepatocytes. In line with our study, a study by Foged et $\mathrm{a}^{41}$ also confirmed that optimal particle diameter for rapid uptake by human immature DCs is below $50 \mathrm{~nm}$. Pathways by which NPs enter cells are to date still not fully understood and depend on cell type, size, surface charge, shape, and bulk material of NPs used. ${ }^{39,42,43}$ Rejman et $\mathrm{al}^{44}$ found that internalization of particles smaller than $200 \mathrm{~nm}$ involves clathrin-mediated endocytosis, while with increasing particle size caveolaemediated internalization occurs. Another study showed that larger PS particles $(200 \mathrm{~nm})$ are found on the cell surface, ${ }^{40}$ suggesting that larger particles could be bound to receptors on the plasma membrane prior to internalization. Moreover, in another study from our group, we employed the J774A.1 mouse macrophage cell line to compare uptake of 1,000 nm and $40 \mathrm{~nm}$ PS particles using different inhibitors of endocytotic pathways (Kuhn et al, unpublished data, 2014). We found that $40 \mathrm{~nm}$ PS particles undergo clathrinmediated uptake and macropinocytosis or phagocytosis, while 1,000 nm PS particles are taken up only by macropinocytosis or phagocytosis.

A salient finding in the present study is that uptake of $20 \mathrm{~nm}$ PS particles by BMDCs was unchanged following LPS exposure, whereas uptake of 1,000 nm PS particles was increased in LPS-exposed BMDCs. These results are contrary to our general understanding that immature DCs are endowed with ability for antigen uptake but with a poor capacity to present antigen to naïve T-cells. In response to antigen encounter or danger signals such as LPS, DCs undergo maturation and are capable to prime naïve T-cells while shutting down their ability for antigen uptake. ${ }^{12,45} \mathrm{~A}$ potential explanation for increased uptake of larger particles may be that immature BMDCs, upon activation by LPS, increase the number of dendrites and therefore their surface area. Larger PS particles may therefore more readily adhere and be taken up by BMDCs. These findings should, however, be further investigated. It was noteworthy, however, that although PS-particle uptake was not affected by LPS stimulation, our data confirmed that LPS exposure and maturation of BMDCs decreased their capacity for uptake of soluble antigen (OVA).

DCs are key players in induction of immune responses, and their function is closely influenced by their level of socalled maturation. Maturation of DCs is known to be mediated by different danger signals such as microbial products and results in upregulated expression of co-stimulatory molecules. ${ }^{46}$ To assess effects of PS-particle incubation on BMDCs, we analyzed BMDC maturation and phenotype after PS-particle treatment. Our data showed that PS-particle treatment did not induce or alter maturation of BMDCs, as frequencies of cells expressing MHC Class II, CD40, and CD86 remained unaffected. Findings reported in the literature regarding capacity of PS particles to induce maturation of immune cells also show that with similar conditions (concentration and incubation time), no significant difference in expression levels of activation markers occurs following incubation with PS particles. ${ }^{20,35}$

Another aspect of DC immune function is the capacity of antigen uptake and antigen degradation. While uptake of labeled OVA protein was not altered by the presence of PS particles, degradation of antigen was downregulated in BMDCs treated with $20 \mathrm{~nm}$ PS particles, but not with $1,000 \mathrm{~nm}$ PS-particle treatment. The model antigen OVA employed in antigen-uptake experiments was endotoxin-free and not modified by the addition of carbohydrate moieties; therefore, as myeloid C-type lectin receptors (CLR) on immune cells such as DCs recognize carbohydrate moieties on antigen, ${ }^{47}$ it is unlikely that CLR-binding of OVA-Alexa647 accounts for the extent of antigen uptake observed. These new findings confirm that particle size may represent a relevant parameter determining DC function, as only BMDCs treated with nanosized particles were unable to fully degrade or process OVA antigen. Interestingly, the capacity of particle-treated BMDCs to stimulate $\mathrm{CD} 4^{+} \mathrm{T}$-cells remained stable when compared with untreated BMDCs, indicating that decreased antigen degradation did not affect induction of T-cell proliferation. Indeed, although antigen degradation capacity was significantly decreased in the present study, it was not completely shut down. We suggest that the reduced level of antigen degradation was still sufficient to induce measurable antigen-specific $\mathrm{CD} 4^{+} \mathrm{T}$-cell proliferation. 
Other in vitro studies using PS particles show divergent findings concerning their immune-modulatory potential. ${ }^{20,35}$ In a recent in vivo study, we showed that negatively charged $20 \mathrm{~nm}$ PS particles are able to enhance antigen presentation to CD4 ${ }^{+} \mathrm{T}$-cells in lung-draining lymph nodes. ${ }^{15}$ Also, another study in vivo demonstrated that glycine-coated PS particles of $50 \mathrm{~nm}$ markedly dampen the ability of CD $11 \mathrm{~b}^{+}$DCs in lungdraining lymph nodes to stimulate OVA-specific T-cells. ${ }^{48}$

In addition to antigen uptake, antigen degradation and T-cell stimulatory capacity, cytokine production in $\mathrm{CD}^{+}$ T-cells co-cultured with particle-exposed BMDCs was assessed. Our study showed no influence of PS particles on expression of all cytokines tested, suggesting that particleexposed BMDCs did not alter the T helper (Th) cell cytokine profiles. Results reported in the literature are discordant on particle-dependent effects on Th profiles. For instance, a Th-1 shift with increased IFN- $\gamma$ production was measured in human immature DCs treated with $75 \mu \mathrm{g} / \mathrm{mL}$ positively charged PS particles, ${ }^{35}$ while an in vivo study showed that PS particles do not induce an increase in BAL fluid IFN- $\gamma$ or IL-17 but prevented Th-2 immunity. ${ }^{48}$ Johrden et al ${ }^{49}$ also demonstrated that production of IFN $-\gamma$ by $\mathrm{CD} 4^{+}$and $\mathrm{CD} 8^{+}$ T-cells is not increased following incubation with PS particles. These findings further reveal that comparability between studies is often limited. Indeed, many parameters may have been an influence when investigating NP potential to modulate immune cells: size, coating, and surface charge of the administered NPs, but also responder T-cell types utilized, concentration of particles, endotoxin contamination, or incubation time.

In search of an explanation for reduced antigen degradation in BMDCs following exposure to $20 \mathrm{~nm}$ PS particles, we detected increased co-localization with lysosomes compared with 1,000 nm PS particles, but without causing measurable changes in lysosomal permeability. Recent studies indicate that NP toxicity is associated with oxidative stress and inflammation, but underlying mechanisms are not known in detail. ${ }^{50-52}$ Among other potential mechanisms, lysosomal dysfunction and impairment of autophagy have been described to be a consequence of NP-cell interactions. ${ }^{53}$ There are different possible ways by which NPs can induce these alterations. It was first shown by several studies that since NPs are sequestered in lysosomes they could accumulate in those compartments and induce lysosomal dysfunction by inducing membrane permeability or defects in lysosomal trafficking. ${ }^{27,29,54}$ We could show that particles were mainly localized in vesicles, including lysosomes, but not in other cellular compartments such as mitochondria or the cell nucleus.
By applying a quantitative analysis by stereology on transmission electron microscopy, which has a much higher resolution, we showed that the preferred particle localization is in vesicles. ${ }^{55}$ Also, it was proposed by Monick et al $^{56}$ that lysosomal overload by particles induces blockade of autophagy flux in alveolar macrophages from smokers, resulting in mitochondrial dysfunction and cytotoxicity. Moreover, overloading lysosomes with indigestible nanomaterials such as gold, was shown to block autophagosome-lysosome fusion and lysosomal degradation. ${ }^{28}$ This finding may therefore indicate that supposedly inert, yet biopersistent nanomaterials such as PS may not cause direct toxicity or cellular effects, but may be of greater concern, as they accumulate in lysosomes where they are not degraded properly and might block other key cellular functions in DC such as antigen degradation.

To further understand possible causes for impaired antigen degradation capacity, we thoroughly investigated potential size-dependent induction of ER stress and expression of pro-inflammatory cytokines by measuring respective mRNA levels of ER stress gene markers GRP78, CHOP, and spliced XBP1 and the pro-inflammatory markers IL-1 $\beta$ and IL-6. Neither particle size was found to induce ER stress or expression of pro-inflammatory cytokine mRNA. As a major indicator of ER stress, GRP78 (78 kDa glucose-regulated protein, also called BiP) is an ER chaperone protein holding stress-sensing proteins in an inactivate state in normal conditions. ${ }^{57}$ Upon ER stress, IRE1, a transmembrane protein, mediates the removal and thus splicing of an intron from XBP1 (x-box binding protein 1) mRNA. This spliced form of XBP1 is then an effective transcription factor that translocates to the nucleus for transcription of various genes such as GRP78. As previously reported, in humans, XBP1 is also required for the transcription of $\mathrm{MHCII}$ genes. ${ }^{58,59} \mathrm{CHOP}(\mathrm{C} /$ EBP homologous protein, also called DNA damage-inducible transcript 3) is involved in ER stress-related apoptosis and is upregulated when there is unsuccessful cell recovery. It has been reported in different studies that the three markers for ER stress response we measured are reliable for the detection of ER stress and that these are upregulated following incubation with silver and poly(lactic-co-glycolic acid) NPs. ${ }^{31,60} \mathrm{We}$ are not aware of any study investigating ER stress during PS-particle exposure. Therefore, we here report for the first time data suggesting that PS particles of either size did not induce ER stress response, inflammation, and apoptosis, contrary to other NP types.

To our knowledge, the underlying study provided initial evidence in the field that nanosized particles accumulate in lysosomes, affecting key lysosomal functions such as antigen 
degradation. Moreover, we also showed that these effects were size-dependent, as only NPs (20 nm) but not microparticles $(1,000 \mathrm{~nm})$ of the same material caused impaired antigen degradation. Therefore, particle size is a relevant parameter determining DC function and may critically influence downstream immune responses. Although other BMDC functions such as antigen uptake or antigen-specific $\mathrm{CD} 4^{+}$ T-cell stimulation remained unaffected, these data highlight the complexity of cellular interactions with supposedly inert, yet biopersistent, nanomaterials causing functional alterations in key immune cells such as DCs through congestion of the lysosomal compartment. It is tempting to speculate that a larger amount of $20 \mathrm{~nm}$ PS particles with a relatively greater surface area might lead to increased absorption of proteolytic enzymes in lysosomes - further investigations will be necessary to understand the mechanisms involved. These findings have important implications for the future design of novel therapeutic nanocarriers that may require either an enhanced or dampened immune response to unfold their clinical effects.

\section{Acknowledgments}

We gratefully acknowledge the expert technical assistance provided by Patrizia Facklam, Dimitri Vanhecke, and Aline Schoegler, as well as Laura Morf for helpful discussions. Microscopy acquisition and analysis were performed with the support of the Microscopy Imaging Center at the University of Berne, Switzerland.

\section{Disclosure}

This study was funded by the Swiss National Science Foundation grants 320030-122355 and NFP64 406440-131266. The authors have no other affiliations or financial involvement with any organization or entity with a financial interest. The authors have no other conflicts of interest in this work.

\section{References}

1. Fadeel B, Garcia-Bennett AE. Better safe than sorry: understanding the toxicological properties of inorganic nanoparticles manufactured for biomedical applications. Adv Drug Deliv Rev. 2010;62(3):362-374.

2. Mahon E, Salvati A, Baldelli Bombelli F, Lynch I, Dawson KA. Designing the nanoparticle-biomolecule interface for "targeting and therapeutic delivery". J Control Release. 2012;161(2):164-174.

3. Xia T, Kovochich M, Liong M, Zink JI, Nel AE. Cationic polystyrene nanosphere toxicity depends on cell-specific endocytic and mitochondrial injury pathways. ACS Nano. 2008;2(1):85-96.

4. Clift MJD, Rothen-Rutishauser B, Brown DM, et al. The impact of different nanoparticle surface chemistry and size on uptake and toxicity in a murine macrophage cell line. Toxicol Appl Pharmacol. 2008;232(3):418-427.

5. Thiele L, Rothen-Rutishauser B, Jilek S, Wunderli-Allenspach H, Merkle HP, Walter E. Evaluation of particle uptake in human blood monocyte-derived cells in vitro. Does phagocytosis activity of dendritic cells measure up with macrophages? J Control Release. 2001;76(1-2):59-71.
6. Petros RA, DeSimone JM. Strategies in the design of nanoparticles for therapeutic applications. Nat Rev Drug Discov. 2010;9(8):615-627.

7. Von Garnier C, Nicod LP. Immunology taught by lung dendritic cells. Swiss Med Wkly. 2009;139(13-14):186-192.

8. Vermaelen K, Pauwels R. Pulmonary dendritic cells. Am J Respir Crit Care Med. 2005;172(5):530-551.

9. Kunda NK, Somavarapu S, Gordon SB, Hutcheon GA, Saleem IY. Nanocarriers targeting dendritic cells for pulmonary vaccine delivery. Pharm Res. 2013;30(2):325-341.

10. Tonnis WF, Kersten GF, Frijlink HW, Hinrichs WLJ, de Boer AH, Amorij J-P. Pulmonary vaccine delivery: a realistic approach? J Aerosol Med Pulm Drug Deliv. 2012;25(5):249-260.

11. Shortman K. Burnet oration: dendritic cells: multiple subtypes, multiple origins, multiple functions. Immunol Cell Biol. 2000;78(2):161-165.

12. Banchereau J, Briere F, Caux C, et al. Immunobiology of dendritic cells. Annu Rev Immunol. 2000;18:767-811.

13. Steinman RM, Banchereau J. Taking dendritic cells into medicine. Nature. 2007;449(7161):419-426.

14. Hancock DG, Guy TV, Shklovskaya E. Experimental models to investigate the function of dendritic cell subsets: challenges and implications. Clin Exp Immunol. 2013;171:147-154.

15. Blank F, Stumbles PA, Seydoux E, et al. Size-dependent Uptake of Particles by Pulmonary APC Populations and Trafficking to Regional Lymph Nodes. Am J Respir Cell Mol Biol. 2013;49:67-77.

16. Lutz MB, Kukutsch N, Ogilvie AL, et al. An advanced culture method for generating large quantities of highly pure dendritic cells from mouse bone marrow. J Immunol Methods. 1999;223(1):77-92.

17. Von Garnier C, Wikstrom ME, Zosky G, et al. Allergic airways disease develops after an increase in allergen capture and processing in the airway mucosa. J Immunol. 2007;179(9):5748-5759.

18. Blank F, Gerber P, Rothen-Rutishauser B, et al. Biomedical nanoparticles modulate specific CD4(+) T cell stimulation by inhibition of antigen processing in dendritic cells. Nanotoxicology. 2011;5:606-621.

19. Schönthal AH. Endoplasmic reticulum stress: its role in disease and novel prospects for therapy. Scientifica (Cairo). 2012;2012:857516.

20. Zupke O, Distler E, Baumann D, et al. Biomaterials preservation of dendritic cell function upon labeling with amino functionalized polymeric nanoparticles. Biomaterials. 2010;31(27):7086-7095.

21. Karlson TDL, Kong YY, Hardy CL, Xiang SD, Plebanski M. The signalling imprints of nanoparticle uptake by bone marrow derived dendritic cells. Methods. 2013;60(3):275-283.

22. Daro E, Pulendran B, Brasel K, et al. Polyethylene glycol-modified GM-CSF expands CD11b high CD11b high but not CD11b low $\mathrm{CD} 11 \mathrm{c}$ high murine dendritic cells in vivo: a comparative analysis with Flt3 ligand. J Immunol. 2000;165:49-58.

23. Autenrieth SE, Autenrieth IB. Variable antigen uptake due to different expression of the macrophage mannose receptor by dendritic cells in various inbred mouse strains. Immunology. 2009;127(4):523-529.

24. Tardif V, Riquelme SA, Remy S, et al. Carbon monoxide decreases endosome-lysosome fusion and inhibits soluble antigen presentation by dendritic cells to T cells. Eur J Immunol. 2013;43(11): 2832-2844.

25. Roederer M. Interpretation of cellular proliferation data: avoid the panglossian. Cytometry. 2011;79(2):95-101.

26. Kopf M, Bachmann MF, Marsland BJ. Averting inflammation by targeting the cytokine environment. Nat Rev Drug Discov. 2010;9(9):703-718.

27. Wu H-Y, Chung M-C, Wang C-C, Huang C-H, Liang H-J, Jan T-R. Iron oxide nanoparticles suppress the production of IL-1beta via the secretory lysosomal pathway in murine microglial cells. Part Fibre Toxicol. 2013;10(1):46.

28. Ma X, Wu Y, Jin S, et al. Gold nanoparticles induce autophagosome accumulation through size-dependent nanoparticle uptake and lysosome impairment. ACS Nano. 2011;5(11):8629-8639.

29. Fröhlich E, Meindl C, Roblegg E, Ebner B, Absenger M, Pieber TR Action of polystyrene nanoparticles of different sizes on lysosomal function and integrity. Part Fibre Toxicol. 2012;9(1):26. 
30. Rocha N, Neefjes J. MHC class II molecules on the move for successful antigen presentation. EMBO J. 2008;27(1):1-5.

31. Christen V, Capelle M, Fent K. Silver nanoparticles induce endoplasmatic reticulum stress response in zebrafish. Toxicol Appl Pharmacol. 2013;272(2):519-528.

32. Adolph T-E, Niederreiter L, Blumberg RS, Kaser A. Endoplasmic reticulum stress and inflammation. Dig Dis. 2012;30(4):341-346.

33. Rodriguez-Lorenzo L, Fytianos K, Blank F, von Garnier C, RothenRutishauser B, Petri-Fink A. Fluorescence-Encoded Gold Nanoparticles: Library Design and Modulation of Cellular Uptake into Dendritic Cells. Small. 2014:1-10.

34. Lehmann AD, Parak WJ, Zhang F, et al. Fluorescent-magnetic hybrid nanoparticles induce a dose-dependent increase in proinflammatory response in lung cells in vitro correlated with intracellular localization. Small. 2010;6(6):753-762.

35. Frick SU, Bacher N, Baier G, Mailänder V, Landfester K, Steinbrink $\mathrm{K}$. Functionalized polystyrene nanoparticles trigger human dendritic cell maturation resulting in enhanced $\mathrm{CD}^{+} \mathrm{T}$ cell activation. Macromol Biosci. 2012;12(12):1637-1647.

36. Fröhlich E. The role of surface charge in cellular uptake and cytotoxicity of medical nanoparticles. Int J Nanomedicine. 2012;7:5577-5591.

37. Liu Y, Li W, Lao F, et al. Intracellular dynamics of cationic and anionic polystyrene nanoparticles without direct interaction with mitotic spindle and chromosomes. Biomaterials. 2011;32(32):8291-8303.

38. Nemmar A, Hoylaerts MF, Hoet PH, Vermylen J, Nemery B. Size effect of intratracheally instilled particles on pulmonary inflammation and vascular thrombosis. Toxicol Appl Pharmacol. 2003;186(1):38-45.

39. Dos Santos T, Varela J, Lynch I, Salvati A, Dawson KA. Quantitative assessment of the comparative nanoparticle-uptake efficiency of a range of cell lines. Small. 2011;7(23):3341-3349.

40. Johnston HJ, Semmler-Behnke M, Brown DM, Kreyling W, Tran L, Stone V. Evaluating the uptake and intracellular fate of polystyrene nanoparticles by primary and hepatocyte cell lines in vitro. Toxicol Appl Pharmacol. 2010;242(1):66-78.

41. Foged C, Brodin B, Frokjaer S, Sundblad A. Particle size and surface charge affect particle uptake by human dendritic cells in an in vitro model. Int J Pharm. 2005;298(2):315-322.

42. Thiele L, Merkle HP, Walter E. Phagocytosis and phagosomal fate of surface-modified microparticles in dendritic cells and macrophages. Pharm Res. 2003;20(2):221-228.

43. Hillaireau H, Couvreur P. Nanocarriers' entry into the cell: relevance to drug delivery. Cell Mol life Sci. 2009;66(17):2873-2896.

44. Rejman J, Oberle V, Zuhorn IS, Hoekstra D. Size-dependent internalization of particles via the pathways of clathrin- and caveolae-mediated endocytosis. Biochem J. 2004;377(Pt 1):159-169.

45. Upham J, Stumbles PA. Why are dendritic cells important in allergic diseases of the respiratory tract? Pharmacol Ther. 2003;100(1):75-87.
46. Blank F, von Garnier C, Obregon C, Rothen-Rutishauser B, Gehr P, Nicod L. Role of dendritic cells in the lung: in vitro models, animal models and human studies. Expert Rev Respir Med. 2008;2(2):215-233.

47. Maglinao M, Eriksson M, Schlegel MK, et al. A platform to screen for C-type lectin receptor-binding carbohydrates and their potential for cell-specific targeting and immune modulation. $J$ Control Release. 2014; $175: 36-42$.

48. Hardy CL, LeMasurier JS, Belz GT, et al. Inert 50-nm polystyrene nanoparticles that modify pulmonary dendritic cell function and inhibit allergic airway inflammation. J Immunol. 2012;188(3):1431-1441.

49. Johrden L, Tenbusch M, Lietz R, et al. Comparison of polystyrene nanoparticles and UV-inactivated antigen-displaying adenovirus for vaccine delivery in mice. Virol J. 2013;10:1-5.

50. Stern ST, Adiseshaiah PP, Crist RM. Autophagy and lysosomal dysfunction as emerging mechanisms of nanomaterial toxicity. Part Fibre Toxicol. 2012;9(1):20.

51. Pelaz B, Charron G, Pfeiffer C, et al. Interfacing engineered nanoparticles with biological systems: anticipating adverse nano-bio interactions. Small. 2013;9(9-10):1573-1584.

52. Li N, Xia T, Nel AE. The role of oxidative stress in ambient particulate matter-induced lung diseases and its implications in the toxicity of engineered nanoparticles. Free Radic Biol Med. 2008;44(9):1689-1699.

53. Levine B. Eating oneself and uninvited guests: autophagy-related pathways in cellular defense. Cell. 2005;120(2):159-162.

54. Lunov O, Syrovets T, Loos C, et al. Amino-functionalized polystyrene nanoparticles activate the NLRP3 inflammasome in human macrophages. ACS Nano. 2011;5(12):9648-9657.

55. Brandenberger C, Mühlfeld C, Ali Z, et al. Quantitative evaluation of cellular uptake and trafficking of plain and polyethylene glycol-coated gold nanoparticles. Small. 2010;6(15):1669-1678.

56. Monick MM, Powers LS, Walters K, et al. Identification of an autophagy defect in smokers' alveolar macrophages. J Immunol. 2010;185(9):5425-5435.

57. Chen R, Huo L, Shi X, et al. Endoplasmic reticulum stress induced by zinc oxide nanoparticles is an earlier biomarker for nanotoxicological evaluation. ACS Nano. 2014;8(3):2562-2574.

58. He Y, Sun S, Sha H, et al. Emerging roles for XBP1, a sUPeR transcription factor. Gene Expr. 2010;15(1):13-25.

59. Iwakoshi NN, Pypaert M, Glimcher LH. The transcription factor XBP-1 is essential for the development and survival of dendritic cells. J Exp Med. 2007;204(10):2267-2275.

60. Hou CC, Tsai TL, Su WP, et al. Pronounced induction of endoplasmic reticulum stress and tumor suppression by surfactant-free poly(lacticco-glycolic acid) nanoparticles via modulation of the PI3K signaling pathway. Int J Nanomedicine. 2013;8:2689-2707. 


\section{Supplementary materials}

Table SI Size measurement of $20 \mathrm{~nm}$ and I,000 nm polystyrene particles employing DLS

\begin{tabular}{|c|c|c|}
\hline $\begin{array}{l}\text { Size } \\
\text { (given by manufacturer) }\end{array}$ & $\begin{array}{l}\text { Diameter/ } \sigma \\
\text { measured by DLS } \\
\text { PBS }\end{array}$ & $\begin{array}{l}\text { Diameter/ } \sigma \\
\text { measured by DLS } \\
\text { IMDM + I0\% FCS }\end{array}$ \\
\hline $20 \mathrm{~nm}$ & $31.3 \mathrm{~nm} / 58.2 \%$ & $43.9 \mathrm{~nm} / 66.4 \%$ \\
\hline $\mathrm{I}, 000 \mathrm{~nm}$ & $941.2 \mathrm{~nm} / 19.6 \%$ & $944.9 / 19.7 \%$ \\
\hline
\end{tabular}

Notes: Measurements were performed in PBS and cell medium (IMDM +10\% FCS). To account for polydispersity, the intensity-weighted statistical average was normalized by particle volume distribution, approximating a number-weighted statistical average of spherical nanoparticles. The diameter represents arithmetic average, and the dispersion in size is quantified by $\sigma$ (polydispersity index = SD/average). The onset of aggregation can be excluded, proved by DLS and visual inspection. Furthermore, the samples were under observation for more than 3 days, during which time the initial stability was preserved.

Abbreviations: DLS, dynamic light scattering; FCS, fetal calf serum; IMDM, Iscove's Modified Dulbecco's Medium; PBS, phosphate-buffered saline; SD, standard deviation.
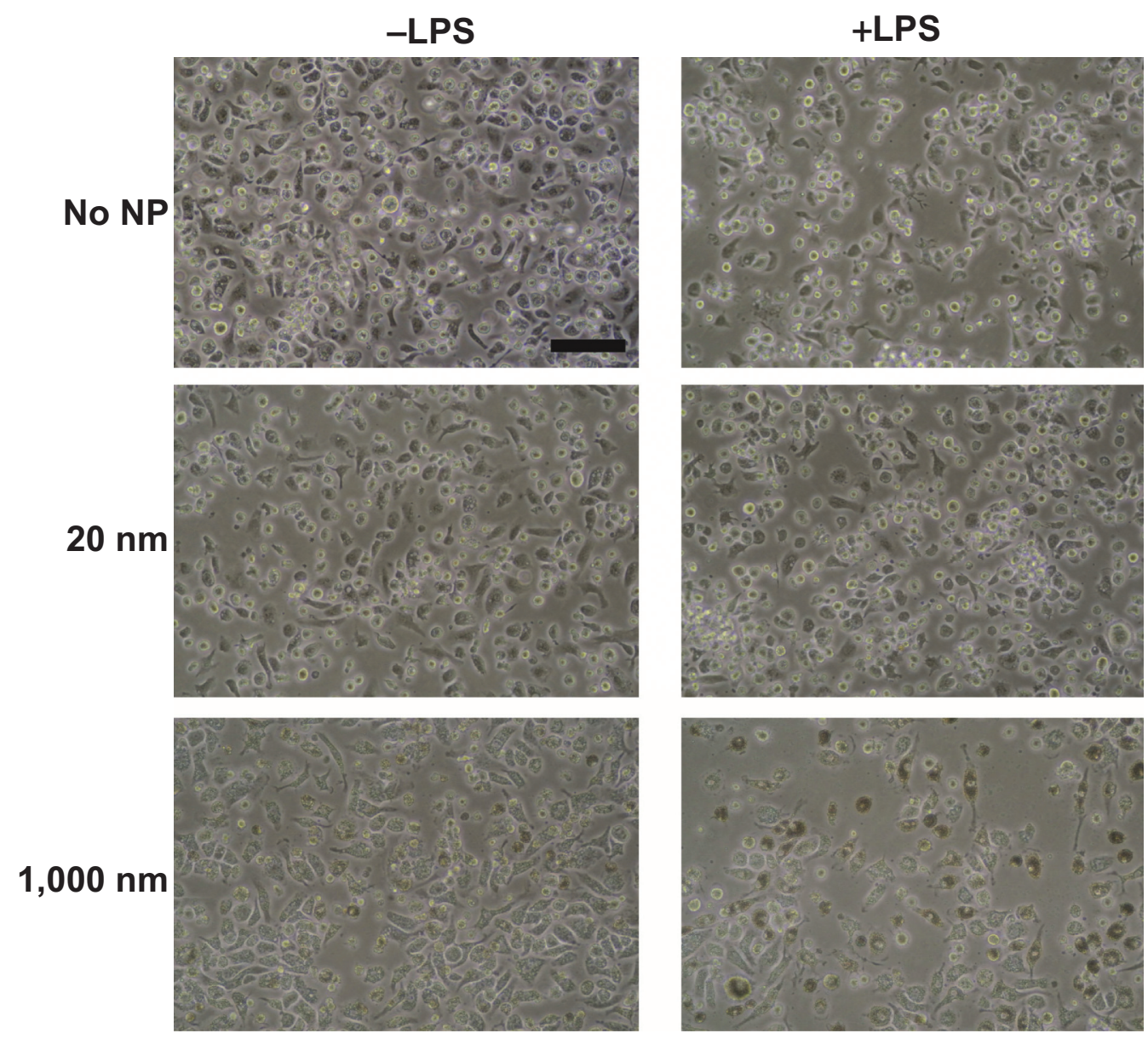

Figure SI Assessment of BMDC viability using light microscopy.

Notes: Images show that BMDC morphology was not altered by $25 \mu \mathrm{g} / \mathrm{mL}$ PS-particle and LPS treatment. Black bar: $100 \mu \mathrm{m}$. Magnification: 20x.

Abbreviations: BMDC, bone marrow-derived dendritic cell; LPS, lipopolysaccharide; NP, nanoparticle; PS, polystyrene. 


\section{$20 \mathrm{~nm}$}

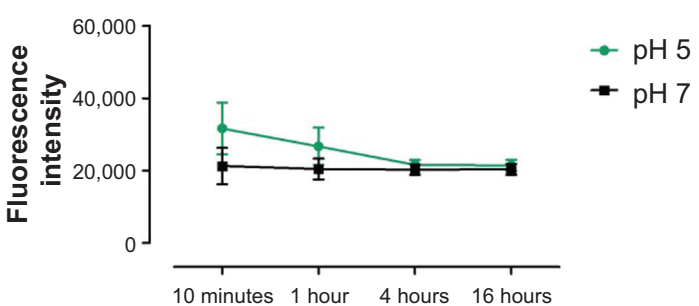

\section{$1,000 \mathrm{~nm}$}

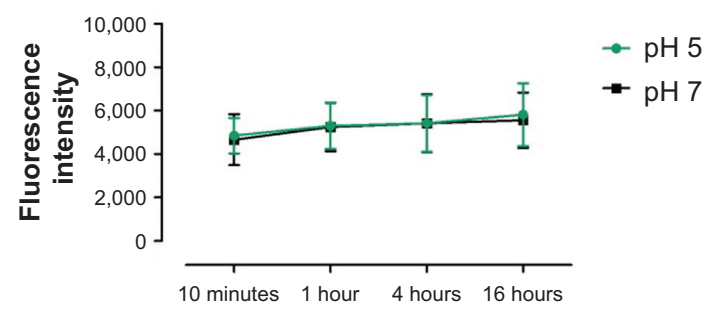

Figure S2 Assessment of emission fluorescence intensity of PS particles in buffer solutions at pH 5 and $\mathrm{pH} 7$ after 10 minutes, I hour, 4 hours, and 16 hours incubation. Notes: Measurements were performed to determine stability of PS-particle fluorescence under physiologic and acidic pH conditions. PS particles were diluted at a concentration of $25 \mu \mathrm{g} / \mathrm{mL}$ in $0.1 \mathrm{M}$ acetate buffer solution at $\mathrm{pH} 5$ and $0.1 \mathrm{M}$ phosphate buffer at $\mathrm{pH}$ 7. Fluorescence intensities were determined at the respective excitation and emission wavelengths specific for each PS particle and given by the manufacturer $(20 \mathrm{~nm}, 505 / 5 \mathrm{I} 5 \mathrm{~nm} ; 1000 \mathrm{~nm}, 44 \mathrm{I} / 486 \mathrm{~nm})$. Results are expressed as $\Delta$ Intensity $=$ measured sample fluorescence intensity minus measured blank (phosphate-buffered saline) fluorescence intensity. Measurements were performed on an Infinite MI000 PRO (Tecan, Männedorf, Switzerland). Three independent experiments were performed $(n=3)$; for each experiment, five measurements with identical parameters were averaged.

Abbreviation: PS, polystyrene.

\section{Publish your work in this journal}

The International Journal of Nanomedicine is an international, peerreviewed journal focusing on the application of nanotechnology in diagnostics, therapeutics, and drug delivery systems throughout the biomedical field. This journal is indexed on PubMed Central, MedLine, CAS, SciSearch ${ }^{\circledR}$, Current Contents ${ }^{\circledR} /$ Clinical Medicine,
Journal Citation Reports/Science Edition, EMBase, Scopus and the Elsevier Bibliographic databases. The manuscript management system is completely online and includes a very quick and fair peer-review system, which is all easy to use. Visit http://www.dovepress.com/ testimonials.php to read real quotes from published authors. 